\title{
Tyrosine phosphorylation-dependent localization of TmaR, a novel $E$. coli polar protein that controls activity of the major sugar regulator by polar sequestration
}

\author{
Tamar Szoke $^{1}$, Nitsan Albocher ${ }^{1 @}$, Sutharsan Govindarajan ${ }^{1,2 @}$, Anat Nussbaum-Shochat ${ }^{1}$ \\ and Orna Amster-Choder ${ }^{1^{*}}$ \\ ${ }^{1}$ Department of Microbiology and Molecular Genetics, IMRIC, \\ The Hebrew University Faculty of Medicine \\ P.O.Box 12272, Jerusalem 91120, Israel \\ ${ }^{2}$ Current address: Department of Biology, \\ SRM University-AP, Amaravati, Andhra Pradesh - 522 502, India \\ ${ }^{\circledR}$ These authors have contributed equally to this work
}

Running title: Tyrosine-phosphorylated TmaR controls sugar metabolism by regulating EI assembly at the pole

*Corresponding Author: ornaam@ekmd.huii.ac.il

Phone: 9722675 8460; FAX: 97226757910

Key words: bacterial polarity / cell pole / proteins localization / dynamic localization / PTS system/ tyrosine phosphorylation 


\section{ABSTRACT}

The poles of $E$. coli cells are emerging as hubs for major sensory systems, but the polar determinants that allocate their components to the pole are largely unknown. Here, we describe the discovery of a novel protein, TmaR, which localizes to the $E$. coli cell pole when phosphorylated on a tyrosine residue. TmaR is shown here to control the subcellular localization of the general PTS protein Enzyme I (EI) by preventing it from exerting its activity by binding and polar sequestration, thus regulating sugar uptake and metabolism. Depletion or overexpression of TmaR results in El release from the pole or enhanced recruitment to the pole, which leads to increasing or decreasing the rate of sugar consumption, respectively. Notably phosphorylation of TmaR is required to release $\mathrm{El}$ and enable its activity. Like TmaR, the ability of El to be recruited to the pole depends on phosphorylation of one of its tyrosines. In addition to hyperactivity in sugar consumption, the absence of TmaR also leads to detrimental effects on the ability of cells to survive in mild acidic conditions. Our results argue that this survival defect, which is sugar- and Eldependent, reflects the difficulty of cells lacking TmaR to enter stationary phase. Our study identifies TmaR as the first $E$. coli protein reported to localize in a tyrosine-dependent manner and to control the activity of other proteins by their polar sequestration and release.

\section{SIGNIFICANCE}

In recent years, we have learnt that bacterial cells have intricate spatial organization despite the lack of membrane-bounded organelles. The endcaps of rod-shaped bacteria, termed poles, are emerging as hubs for sensing and responding, but the underlying mechanisms for positioning macromolecules there are largely unknown. We discovered a novel protein, TmaR, whose polar localization depends on a phospho-tyrosine modification. We show that TmaR controls the activity of $\mathrm{El}$, the major regulator of sugar metabolism in most bacteria, by polar sequestration and release. Notably, TmaR is essential for survival in conditions that $E$. coli often encounters in nature. Hence, TmaR is a key regulator that connects tyrosine phosphorylation, spatial regulation, sugar metabolism and survival in bacteria and the first protein reported to recruit proteins to the $E$. coli cell poles. 


\section{INTRODUCTION}

The central dogma describes the flow of genetic information from DNA to RNA to protein. However, for this process to be successful, the final product - the protein - needs to be in the right place, where it is required, and at the right time. The consequences of mislocalization can be harmful to any cell type, let alone to the unicellular bacterial cell, whose survival depends on fast and efficient response to environmental changes. Hence, protein localization is an important posttranslational regulatory step. Thus far, most examples of protein targeting were reported in eukaryotic cells, usually in the context of transport from one organelle to another (1). In recent years, it became evident that localization of both proteins and RNAs to specific subcellular domains occurs also in prokaryotic cells, and is vital for many cellular processes (2-5). However, the mechanisms underlying macromolecules targeting to specific subcellular domains in bacterial cells, with the exception of membrane and cell division proteins, remain largely unknown.

The bacterial cell poles are emerging as important domains that accommodate protein and RNA assemblies $(5,6)$. Pole-localized proteins are involved in a wide range of cellular functions, including motility, regulation of cell cycle, metabolism, differentiation, pathogenesis and secretion (7). Various proteins are kept at the E. coli cell pole as inactive till they are needed, e.g., MurG (8), and FtsZ (9). On the other hand, most proteins of the chemotaxis complex act at the pole, except for CheY that is activated at the pole, but leaves to exert its effect on the flagella rotors located around the cell circumference (10). The phosphotransferase system (PTS), which controls sugar utilization and metabolism in most bacteria, provides another example for regulation via polar cluster formation. Execution of the PTS functions depends on a phosphorylation cascade that initiates with EI and HPr - the general PTS proteins - that deliver the phosphate to the PTS sugar permeases, which import and phosphorylate the incoming sugars (11). The PTS proteins also exert different effects on non-PTS proteins depending on their phosphorylation on histidine residues, thus modulating the hierarchy in sugar utilization (11). The PTS-imported sugars enter glycolysis, whose product, phosphoenolpyruvate (PEP), phosphorylates El, making El an important link between glycolysis and sugar uptake (12). We have previously shown that the general PTS proteins localize to the E. coli cell poles (13), although their localization depends on yet unknown factors (14), that during growth, El polar clusters form stochastically from pre-existing dispersed molecules, and that El clustering negatively correlates with El function (15). Still, conclusive proof for polar localization as an inhibitory mechanism of El function is lacking and the identity of the factor that captures $\mathrm{El}$ at the pole remained unknown.

Polar clusters offer additional benefits, such as communication between signal transduction systems in order to generate an optimal response, e.g., the chemotaxis and the PTS system (16), or the establishment of cellular asymmetry to coordinate developmental programs with cell-cycle progression (7). Polar proteins that recruit other proteins to the poles, thus regulating cell cycle progression, were discovered in some bacteria, e.g., DivIVA in Bacillus subtilis, PopZ and TipN in 
Caulobacter crescentus and HubP in Vibrio cholerae (7). However, no pole-localizing protein has been identified in $E$. coli thus far.

In some bacteria, e.g., C. crescentus, specific localization of proteins is linked to their phosphorylation or to the phosphorylation of factors regulating them $(17,18)$. In most cases, these proteins are members of the two-component systems, which mediate sensing and regulation by phosphorylation on histidine and aspartic acid residues (19). These phosphorylation events have been considered as most prevalent in bacteria. Only in recent years, the combination of improved methodologies revealed numerous previously unknown Ser/Thr/Tyr phosphorylation sites, once thought to be hallmarks of phosphorylation in eukaryotes, in bacterial and archaeal proteins. The degree to which these putative sites are phosphorylated is still unclear and proofs for their roles and importance in vivo are just beginning to emerge. Also, the linkage between phosphorylation on Ser/Thr/TyR and localization of the phosphorylated bacterial proteins remained unknown.

In this study, we show that a previously uncharacterized E. coli protein, YeeX, which is prevalent among Gram-negative bacteria, clusters at the pole in a tyrosine phosphorylation-dependent manner and recruits the major sugar utilization regulator EI. We, therefore, renamed this protein TmaR for targeting of sugar metabolism-associated regulator. TmaR and El are shown to physically interact and to co-localize. TmaR is necessary for El polar clustering, but the opposite is not true. Only phosphorylated TmaR can release El from the poles, since the diffuse nonphosphorylated TmaR binds to El quite irreversibly. Notably, tyrosine phosphorylation of El is also requires for its polar localization. We further show that TmaR-mediated El clustering inversely correlates with El-mediated sugar uptake, implying that the polar clusters serve as a reservoir for ready-to-act EI molecules. Cells lacking TmaR have detrimental effects on cell survival in an EI concentration- and sugar concentration-dependent manner when challenged with mildly acidic conditions, typical to various $E$. coli habitats. Taken together, our study identifies TmaR as a spatial regulator of sugar metabolism and bacterial survival and as the first reported $E$. coli polelocalizer.

\section{RESULTS}

\section{TmaR assembles into polar clusters in a tyrosine phosphorylation-dependent manner}

To identify yet unexplored polar proteins, we screened by fluorescence microscopy the library constructed by Xie and coworkers of 1018 E. coli genes tagged with YFP (20). This library allows the observation of C-YFP-tagged proteins expressed from their corresponding loci and native promoters in the chromosome. We thus identified TmaR (previously designated YeeX), a 109 residue-long protein of unknown function predicted to form a coiled-coil structure (https://zhanglab.ccmb.med.umich.edu/QUARK/), which is conserved among Gram-negative bacteria (see SI Appendix, Fig. S1). The TmaR-YFP protein formed clusters that localized mainly near one pole of the rod-shaped E. coli cells (Fig. 1A). Measurements of the YFP intensity along 
the long axis of clusters-containing cells demonstrated that the vast majority of these cells had a peak in one pole (Fig. 1B), indicating that they have a TmaR-YFP cluster localizing to one of the poles. To verify that this localization is not due to the fluorescent tag and is not affected by the site of fusion, we monitored the localization of a TmaR that was $\mathrm{N}$ terminally tagged with monomeric YFP and expressed from its native chromosomal locus and promoter. The results (see SI Appendix, Fig. S2) show that TmaR forms polar clusters regardless of the type of tag and of which terminus is tagged.

To further characterize TmaR subcellular localization, we calculated the percentage of cells with detectable TmaR clusters in the population during growth. The results show that in LB, nearly $80 \%$ of cells had detectable TmaR clusters at their pole in early logarithmic (log) phase. This fraction dropped to $25 \%$ in mid-log phase and remained constant during stationary phase (SP) (Fig. 1C). To find out if the change in the fraction of cluster-harboring cells is due to a change in TmaR cellular level or to relocation of pre-existing TmaR molecules, we compared the amount of TmaR in cells at different growth stages by Western blot analysis. The results in Fig. 1D and its quantification in (see SI Appendix, Fig. S3) show that the amount of TmaR does not change much among the different growth phases. This result indicates that the growth phase-dependent difference in the percentage of cells with detectable clusters is due to a change in the distribution pattern of the TmaR molecules, i.e., whether they are assembled into clusters or diffuse in the cytoplasm, and not due to changes in TmaR cellular level.

Our studies of TmaR encouraged us to search the website for any piece of information regarding this protein. Interesting, we found that TmaR is phosphorylated on a tyrosine residue (http://dbpsp.biocuckoo.org/). This database documents the results of a high throughput analysis of the E. coli phosphoproteome, which identified TmaR as phosphorylated on tyrosine 79 (21). Because TmaR has only 3 tyrosines in positions 55, 72 and 79 , we mutated each to a phenylalanine, which is the most similar amino acid to tyrosine, but cannot be phosphorylated. We thus generated strains, expressing YFP-tagged TmaR variants, Y79F, Y72F or Y51F, from their endogenous gene locus on the chromosome. Using fluorescence microscopy, we observed YFPtagged TmaR Y79F and TmaR Y51F at the bacterial cell poles, similar to wild-type TmaR-YFP (Fig. 1E). When calculating the fraction of cells with a polar cluster in each of these three strains (WT and two mutants), no significant change was observed (Fig. 1F), indicating that the two mutants behave precisely like WT. Contrarily, the YFP-tagged TmaR Y72F mutant did not localize to the poles and was completely spread within the cytoplasm (Fig. 1E). Moreover no polar clusters were observed in cells expressing the YFP- tagged double mutant TmaR Y72F,Y79F or the triple mutant TmaR Y79F,Y72F,Y51F, just like in cells expressing the single mutant TmaR Y72-YFP (Fig. 1E and 1F). Taken together, these results suggest that phosphorylation of TmaR on Tyr72 is a prerequisite for its polar localization.

To substantiate the above results, we monitored the localization of monomeric YFP-tagged TmaR variants and obtained similar results, i.e., wild-type TmaR and the Y79F and Y51F TmaR mutants 
were able to form polar clusters, while TmaR Y72F and the double and triple mutants were diffused in all cells (see SI Appendix, Fig. S4). To verify that the differences detected in subcellular localization is not due to a change in the cellular level of TmaR caused by the mutations, we compared the amount of mYFP-TmaR and its tyrosine mutants by Western blot analysis (similar amounts of lysates were analyzed, see SI Appendix, Fig. S5, lower row). The results show that the amount of TmaR does not change in the mutants (see SI Appendix, Fig. S5, upper panel). Finally, although there is no good mimic for phosphorylated tyrosine (pTyr), we mutated Tyr72 to glutamic acid (TmaR Y72E) or to aspartic acid (TmaR Y72D), which are both negatively charged and can partially mimic phosphorylation of other residues (22). The resultant mutant proteins behaved like the phenylalanine substitution, that is, they did not cluster at the pole (see SI Appendix, Fig. S6). This was expected, because, in terms of charge strength and geometry, glutamate and aspartate are not a good mimic for a constantly phosphorylated tyrosine (23). We therefore, continued to work with the Tyr to Phe substitutions, since they are supposed to have a minimal effect on the protein structure.

To get a direct proof for TmaR phosphorylation on tyrosine 72, we compared the amount of tyrosine-phosphorylated TmaR and its mutants by Western blot analysis using anti-pTyr antibody. All TmaR variants were expressed from a plasmid, because their level, when expressed from their endogenous gene locus, did not allow their unambiguous detection by this antibody and similar amounts of lysates were analyzed (see SI Appendix, Fig. S7). The results in Fig. 1G show that the three polarly localized TmaR proteins - WT, Y79F and Y51F - were nicely detected by the anti-pTyr antibody, whereas the three diffused TmaR proteins - Y72F and the double and triple mutants showed a very faint band, frequently observed with this antibody due to phosphorylation on other residues (24). These results prove that Tyr72 of TmaR gets phosphorylated and, together with the imaging results, indicate that only the phosphorylated form of TmaR forms polar clusters.

Next, we aimed at identifying the kinase that controls TmaR polar localization by tyrosine phosphorylation. Two bacterial tyrosine kinases (BY kinases), Wzc and Etk, were identified in $E$. coli, the first involved in exopolysaccharide production and the second in capsule assembly (25, 26) . To inquire whether one of these kinases phosphorylates TmaR, we transduced $\Delta w z C$ (Wzc$\mathrm{KO}$ ) or $\Delta y c c C$ (Etk-KO) to our TmaR-YFP-expressing strain. No change in TmaR polar localization was observed as a result of either tyrosine kinase absence (see SI Appendix, Fig. S8). Notably, no reduction in the cellular level of tyrosine phosphorylation was detected in a strain deleted both wzc and etk genes, indicating that $E$. coli contains yet unknown tyrosine kinases (27). Unless TmaR is phosphorylated by both Wzc and Etk, one of the unknown kinases is expected to phosphorylate TmaR.

\section{TmaR and the general PTS protein EI exhibit co-localization and co-dynamics and are capable of interacting with each other}

The general PTS protein El initiates the phosphorylation cascade that controls hierarchical uptake and utilization of preferred carbohydrates from complex environments (28). The soluble El protein 
has previously been shown in our lab to shift between assembling into polar clusters and diffusing in the cytoplasm (13-15), but the factor responsible for its polar localization remained unknown $(13,14)$. We, therefore, asked whether TmaR is involved in determining the subcellular localization of El. Imaging cells, which express TmaR-YFP and El-mCherry expressed from their respective native promoters and loci on the chromosome, by fluorescence microscopy demonstrated that the polar clusters of the two proteins co-localize (Fig. 2A).

Although El-mCherry activity has been demonstrated previously (13) and shown to be comparable to that of wild-type El (15), not only did we verify this again (see SI Appendix, Fig. S9), but we also asked if El-mCherry fluorescent signal correlates with El-mCherry level. To this end, we expressed El-mCherry from a plasmid by increasing concentrations of inducer and measured the mean intensity of the El-mCherry fluorescent signal by imaging (see SI Appendix, Fig. S10A), as well as the protein level using Western blot analysis (see SI Appendix, Fig. S10B,C). These results show that the fluorescence intensity of El-mCherry is proportional to the level of the El protein. Of note, a higher fraction of El-mCherry diffuse molecules was detected when it was co-expressed with nontagged TmaR compared to when it was co-expressed with TmaR-YFP (see SI Appendix, Fig. S11A). However, the fraction of cells with a polar El-mCherry cluster in populations expressing YFP-tagged TmaR is only slightly lower than when expressed with non-tagged TmaR (see SI Appendix, Fig. S11B). Although these differences are minor and are not expected to affect our conclusions, we refrained from observing El localization in the presence of fluorescently-tagged TmaR, unless we aimed at exploring their colocalization.

To assess the degree of El and TmaR co-localization, we inspected the localization of El-mCherry and TmaR-YFP along the long cell axis in 100 cells that contained clusters of both proteins, which represented $63 \%$ of the population. The results, presented as a heat map in Fig. 2B, show that the maximal fluorescence of the two proteins overlaps in all cells and that the two proteins mostly localize to one pole, indicating co-localization of TmaR and El clusters. Because the clusters of both proteins exhibited dynamics, mainly within the pole region, we asked whether they move together or independently. The spatiotemporal behavior of co-expressed YFP-TmaR and EImCherry over time, recorded by time-lapse microscopy, indicated that the dynamics of the two proteins is coordinated, as shown by the kymograph in Fig. 2C. To study the co-occurrence of TmaR and El clusters, we spotted diluted overnight culture of cells expressing YFP-TmaR and EImCherry on an agar pad with fresh medium and monitored the existence of TmaR and El clusters in 163 cells every 3 minutes for 3 hours by time-lapse microscopy. The vast majority of the cells divided once during this period (growth in agar chambers of minimal media without aeration is rather slow) and images of both daughter cells were analyzed, culminating in a final population of about 320 cells that were finally analyzed. The results, presented in Fig. 2D, can be summarized as following: $79 \%$ of the cells contained both TmaR and El clusters, approximately two thirds contained clusters of the two proteins throughout their lifetime, and the rest contained clusters of both at a certain point during their life. Notably, none of the cells in the population contained an EI 
cluster without a TmaR cluster at any point, whereas $15 \%$ of the cells contained only a TmaR cluster sometime during their lifetime. The remaining $6 \%$ did not contain any cluster at any time. These results imply that TmaR clusters in the vast majority of $E$. coli cells, throughout their lifetime or temporarily, with a significant fraction of the cells having also an accompanying El cluster.

Co-localization and co-dynamics of TmaR and EI raised the possibility that the two proteins interact with each other. To check this possibility, we asked if TmaR and El can be co-purified from cells. To this end, we lysed cells expressing or not endogenously expressed TmaR-YFP and incubated the lysates with anti-GFP antibody-bound beads. The proteins that bound to the beads from each strain were eluted and subjected to mass spectrometry analysis, and the fold change of YFP-TmaR binding intensity compared to the non-tagged TmaR intensity was calculated for each protein. El was detected as co-purifying with TmaR at a high (6.3) fold change (Fig. 2F). When plotting the number of peptides versus the log fold change for all the proteins detected in the mass spec analysis (Fig. 2G), El comes up as a bona fide interactor. A Gene Ontology (GO) analysis of the mass spec results identified the biological processes that TmaR-interactors are involved in (Fig. 2H). Notably, the biggest group of TmaR partners is of proteins involved in metabolic processes, raising the possibility that by localizing El, TmaR is involves in its regulation. Of note, another big group of TmaR partners is of proteins that are associated with localization.

Because co-purification of TmaR and El might have been due to indirect interaction, we used the Far-Western technique to ask whether the two proteins can interact directly. To this end, lysates of $\Delta t m a R$ cells expressing His-tagged TmaR from a plasmid (Fig. 2E, lanes 2) and a control lysate of $\Delta t m a R$ cells expressing only the His tag (Fig. 2E, lanes 1) were subjected to SDS-PAGE and blotted onto a nitrocellulose membrane. Three identical membranes (I-III) with identical amount of the two lysates were prepared and probed with anti-His antibody (Western, Fig. 2E, panel I) or with purified El-mCherry or mCherry, followed by antibodies against mCherry (Far-Western, Fig. 2E and see SI Appendix, Fig. S12, panels II and III). The Far-Western results show that EI-mCherry, but not mCherry, interacted with TmaR-His, indicating that TmaR and El have the capacity to interact directly. Of note, the faint band that co-migrates with TmaR, observed in all lanes in membranes II and III, is a non-specific band, as it appears regardless of the lysate analyzed, and it could be explained by the use of polyclonal antibody or by non-specific interaction with mCherry.

Together the results in this section suggest that TmaR and EI co-assemble at the cell pole.

\section{TmaR controls El polar localization, but not vice versa}

The results in the previous section indicate that the subcellular distribution of TmaR and El is coordinated. To test whether one of the two proteins affects localization of the other or their localization is co-dependent, we first observed the localization of El-mCherry in wild-type (WT) and $\Delta t m a R$ (TmaR-KO) strains, as well as in a strain overexpressing TmaR (TmaR-OE). Deletion of the tmaR gene had a dramatic effect on El localization pattern, that is, the cells contained no detectable clusters and El-mCherry was observed as homogenously diffused in the cytoplasm 
(Fig. 3A and 3B, compare WT and TmaR-KO), indicating that El polar localization depends on TmaR. To substantiate that TmaR is the cause for polar localization of El clusters, we complemented the TmaR-KO strain by expressing TmaR from a plasmid and monitored ElmCherry localization. The results demonstrated that polar localization of El clusters was restored (Fig. 3A, TmaR-KO+OE). Of note, the population of wild-type cells overexpressing TmaR contained twice the number of cells with El-mCherry polar clusters in comparison to the population of cells expressing TmaR from the chromosome (Fig. 3B, compare WT and TmaR-OE). Moreover, a comparison of the sum intensity of the El-mCherry clusters fluorescent signals suggested that the average El-mCherry cluster in the TmaR-OE strain contains significantly more El-mCherry molecules than the strain expressing TmaR from the chromosome (Fig. 3C). These results imply that the ability of El to localize to the pole depends entirely on TmaR and the extent of El recruitment to the pole correlates with the cellular level of TmaR.

The observed differences in EI-mCherry localization in the WT, TmaR-KO and TmaR-OE strains is not due to differences in El cellular level, as indicated by the Western blot analysis results presented in Fig. 3D, which show that the three strains contain comparable amounts of ElmCherry. Hence, pre-existing El molecules can join the cluster or diffuse out of it.

Next, we asked whether polar localization of TmaR depends on El localization by observing TmaRYFP expressed from the chromosome of cells deleted for the pts operon that encodes EI (PTS$\mathrm{KO}$ ). The results in Fig. 3E show that TmaR was not affected by the absence of El, as localized to the pole of these cells. Furthermore, we did not observe a significant change in the fraction of cells with TmaR clusters between the PTS-KO and the wild-type strains (Fig. 3F). Hence, polar localization of El requires TmaR, but the opposite is not true. Still, in cells overexpressing El from a plasmid, the chromosomally-encoded TmaR became more diffused (Fig. 3G). The effect of expressing El at an artificially high concentration on TmaR distribution, which can be described as in vivo titration, supports the notion of physical interaction between the two proteins.

El is the first general PTS protein, which initiates the cascade that controls carbohydrate utilization by phosphorylating the second general PTS protein, HPr. Although both El and HPr exhibit polar localization, they were shown to localize to the poles independently (13). We, therefore, asked whether TmaR also controls HPr polar localization. The results in Fig. $\mathbf{3 H}$ show that polar localization of HPr-GFP was not affected when expressed in a TmaR-KO background, indicating that TmaR specifically controls El, but not HPr localization.

\section{TmaR-mediated El localization affects sugar uptake by the PTS system}

The results presented above show that TmaR has a crucial role in El polar clusters localization, raising the possibility that it affects EI function in PTS sugars uptake. To test this proposition, we first streaked TmaR-WT, TmaR-KO and TmaR-OE strains on MacConkey plates supplemented with different PTS sugars, with a PTS-KO strain serving as a negative control. Fig 4A shows growth of these strains on a fructose-supplemented plate, but similar results were observed with 
MacConkey plates supplemented with glucose, mannitol or N-acetylglucosamine (NAG). In all cases was the red color of the colonies of the TmaR-KO strain more intense than that of the WT colonies, and the red color of the WT deeper than that of the TmaR-OE strain. These results suggest that the absence of TmaR makes cells hyper-active for PTS sugar consumption, while overexpression of TmaR reduces PTS activity.

Because TmaR-YFP was used for the TmaR-El localization and interaction studies, we verified that fluorescently tagging TmaR does not affect El function by streaking cells expressing TmaRYFP, mYFP-TmaR or untagged TmaR on a fructose-supplemented MacConkey plate. The results show that El is similarly active in the three strains (see SI Appendix, Fig. S13).

To quantify the effect of TmaR on El function, we compared the ability of strains expressing TmaRWT, TmaR-KO and TmaR-OE to consume glucose by monitoring the drop in glucose concentration in the medium over time (see Supporting Materials and Methods). The results in Fig. 4B verified that the TmaR-KO strain consumes glucose faster than the WT strain, which consumes glucose faster than the TmaR-OE strain. Comparison of the average consumption rate of the three strains corroborated that TmaR-KO consumes glucose two times faster than the WT and TmaROE strains (Fig. 4C). Hence, the activity of El inversely correlates with the level of TmaR in the cell. These results strengthen our previous results, which suggested that clustering of El negatively correlates with El activity (15).

The increased sugar consumption by the $\Delta t m a R$ mutant could be caused by increased availability of El or it could be caused by increased levels of the glucose transporter protein PtsG (EIICB ${ }^{\text {Glc }}$ ), shown before to affect glucose consumption (29). To discriminate between these possibilities, we compared the amount of PtsG in WT and TmaR-KO cells by Western blot analysis, using antibodies against the cytoplasmic C-terminal domain of PtsG (anti-IIB ${ }^{\text {Glc }}$ antibodies, (30)). We saw no difference in the level of PtsG between the two strains, which was lower than PtsG level in cells overexpressing it from a plasmid (see SI Appendix, Fig. S14). These results support the notion that the increase in sugar consumption in cells lacking TmaR is due to an increase in El activity.

An additional assay that we used to compare the effect of TmaR subcellular level on PTS sugars uptake relied on the use of a strain expressing mCerulean, a cyan fluorescent protein (CFP), from the $\mathrm{N}$-acetyl glucosamine (NAG) promoter $\left(\mathrm{P}_{N A G}\right)$ and El-mCherry (31). NAG has been used in the past as a representative of PTS sugars, whose utilization is positively regulated by $\mathrm{El}$, since induction of $P_{N A G}$ by NAG is consistent with the positive feedback mechanism suggested for promoters of PTS sugar utilization genes (31)(15). Hence, production of mCerulean from $P_{\text {NAG }}$ implies that $\mathrm{El}$ is active and enables estimation of $\mathrm{El}$ activity by fluorescence microscopy. After transducing the TmaR-KO mutation into the El-mCherry and Pnag-mCerulean expressing strain, the single cell fluorescence mean intensity of mCerulean and of El-mCherry, which report on EI activity (see SI Appendix, Fig. S10) and on El cellular level, respectively, were plotted against each other for 103 TmaR-WT cells and 103 TmaR KO cells grown in minimal medium containing NAG. The results in Fig. 4D show lack of correlation between the amount of El and its activity in 
the TmaR-WT cells, whereas the level of El in the TmaR-KO strain correlated with its function, as indicated by the Pearson correlation scores $(p)$ and the $r$ squares $\left(R^{2}\right)$. These results suggest that in the strain expressing wild-type TmaR, the amount of active El does not correlate with its cellular level, but rather with the fraction that is not sequestered at the pole. Conversely, in the TmaR-KO strain, in which all El molecules are diffuse, the activity of El correlates with the level of El molecules in the cell. These results are in agreement with the sugar consumption assay (Fig. 4B), which showed that El activity inversely correlates with TmaR level. When presenting the fluorescence mean intensity of mCerulean by a box plot, the level of this protein production in TmaR-KO cells emerges as more heterogeneous than in WT cells (see SI Appendix, Fig. S15), suggesting that not only does TmaR control El activity, but it also limits the degree of heterogeneity in its activity, a phenomenon that has been studied in our lab (15). This implies that flooding the cells with excess of El, which allows irregular level of sugar consumption, disturbs the cells sense of balance and leads to higher fluctuations in sugar uptake.

Overall, the results in this section demonstrate that there is a connection between El localization and function. That is, in the absence of TmaR, El is diffuse and, therefore, more active in sugar uptake, whereas upon TmaR expression, a fraction of El assembles into polar clusters and, therefore, less sugar is taken by the cells. Moreover, our results hint at the possibility that TmaRmediated El assembly controls the population heterogeneity with respect to sugar consumption.

\section{Tyrosine phosphorylation of TmaR is required for the release of El from the poles and for its activity}

The results described thus far, which show that TmaR controls El localization and function and has the potential to interact with El, made us wonder whether TmaR phosphorylation on Tyr72, which is required for its polar localization, affects its ability to interact with El and to control El localization and function.

We first tested if El interaction with TmaR depends on TmaR tyrosine phosphorylation using the Far-Western technique. To this end, similar amounts of lysates ((see SI Appendix, Fig. S16A) of $\Delta$ tmaR cells expressing wild-type TmaR, the single mutants in TmaR tyrosines (Y79F and Y51F), the double mutant or the triple mutant (see above), all from plasmids as His-tagged proteins, as well as lysates of $\Delta t m a R$ cells expressing only a His-tag or no protein from plasmids as controls, were subjected to SDS-PAGE. The proteins were blotted onto a nitrocellulose membrane, incubated with purified mCherry-tagged El, and then with antibodies against the mCherry. The FarWestern results (Fig. 5Ab), before and certainly after quantification by normalizing them to the Western results (Fig. 5Aa and $\mathbf{5 c}$ ), clearly show that although El can interact with all TmaR variants, the interaction with TmaR proteins that lack Tyr72 (non-phosphorylated) is significantly stronger. These results indicate that El affinity for unphosphorylated TmaR is much higher than its affinity for phosphorylated TmaR.

The increased binding of non-phosphorylated TmaR to El raised the question whether TmaR phosphorylation on Tyr72, which prevents its polar clustering, affects El localization. Using 
fluorescence microscopy, we monitored El-mCherry localization in the presence of plasmidexpressed wild-type GFP-TmaR or GFP-TmaR Y72F. Surprisingly, El was recruited to the poles in both strains (Fig. 5B, left panels). Moreover, a portion of the GFP-TmaR Y72F mutant, clustered (Fig. 5B, middle panels) and co-localized with El-mCherry (Fig. 5B, right panels), raising the possibility that the strong binding of $\mathrm{El}$ to non-phosphorylated TmaR led to their co-aggregation and the formation of inclusion bodies. To test this hypothesis, we expressed IbpA, a reporter for inclusion bodies presence (32), tagged with msfGFP, together with El-mCherry, both from the chromosome, and non-tagged TmaR or TmaR Y72F, both from a plasmid. The results show that IbpA--msfGFP formed polar foci that overlapped with El-mCherry in cells expressing nonphosphorylated TmaR, as opposed to IbpA-msfGFP diffuse distribution in the presence of wildtype TmaR (Fig. 5C), indicating that El aggregates to form inclusion bodies only in the presence of non-phosphorylate TmaR. The quantitative significance of this result is shown in SI Appendix, Fig. S17.

Taken together, the results thus far indicate that El binding to TmaR is reversible as long as TmaR can get phosphorylated, whereas its binding to non-phosphorylated TmaR is very strong and quite irreversible. Hence, only phosphorylated TmaR, which recruits El to the poles, can release El from the polar clusters.

Next, we asked if the function of El in sugar uptake is affected by its inability to be released from the poles when TmaR cannot get phosphorylated. To test this, we streaked $\Delta t m a R$ strains harboring plasmids expressing wild-type TmaR, the three single substitution of TmaR tyrosines, and the triple replacement of all TmaR tyrosines, as well as the $\Delta t m a R$ and $\Delta p t s$ controls, on a fructose-supplemented MacConkey plate. The results in Fig. 5D show that only TmaR proteins that cannot get phosphorylated (the double and the triple mutant) are defective in sugar utilization (white colonies), whereas TmaR proteins that can get phosphorylated (WT, Y79F and Y51F) can utilize the sugar (red colonies). These results indicate that overexpression of non-phosphorylated TmaR proteins (lack Y72 residue), which results in irreversible binding of EI and aggregation, prevents El-mediated sugar consumption. Of note, when strains expressing the above TmaR proteins from tmaR native promoter and locus on the chromosome where streaked on a similar plate, all TmaR mutant strains demonstrated a certain ability to consume sugar, although less than TmaR WT strain (see SI Appendix, Fig. S18). Hence, native expression level of nonphosphorylated TmaR leave a certain fraction of El molecules unbound and, hence, active; however, when overexpressed, nonphosphorylated TmaR binds an increased fraction of El and aggregates with it, thus preventing sugar consumption.

\section{Tyrosine phosphorylation of El is important for its polar localization, but not for its function}

Besides its well-known self-phosphorylation on His 189, a high throughput analysis (http://dbpsp.biocuckoo.org/) identified $\mathrm{El}$ as a protein that undergoes phosphorylation on a tyrosine residue, Tyr122 (27), which is a conserved residue (33). To examine if Tyr122 is important for El polar localization, we mutated this tyrosine to phenylalanine and compared the cellular 
distribution of EI Y122F to that of wild-type El, after tagging both proteins by mCherry. The results in Fig. 5E show that unlike El-mCherry, which was detected in polar clusters, EI Y122F-mCherry was completely diffused throughout the cytoplasm. Notably, EI Y122F does not affect TmaR localization (see SI Appendix, Fig. S19), as expected from the results reported above, which demonstrated that $\mathrm{El}$ is not required for TmaR polar localization. The two known tyrosine kinases of $E$. coli do not seem responsible for El phosphorylation, since transduction of a strain expressing El-mCherry with deletions of their genes (Wzc-KO or Etk-KO) did not affect El localization (see SI Appendix, Fig. S20). Hence, the phosphorylated tyrosine in position 122 of El is important for its polar localization.

Since TmaR is important for El recruitment to the poles, we asked whether EI Y122F non-polar localization is due to its inability to interact with TmaR. Using the Far-Western technique, we tested the ability of EI Y122F to interact with TmaR. For this purpose, similar amounts of lysates of $\Delta t m a R$ cells expressing His-tagged TmaR or only a His-tag, were subjected to SDS-PAGE. The proteins were blotted onto two nitrocellulose membranes that were incubated either with anti-His antibodies (Western, Fig. 5F, left panel) or with purified El Y122F-mCherry followed by antimCherry antibodies (Far Western, Fig. 5F, right panel). The results show that the Y122F mutation in El did not eliminate the protein capacity to interact with TmaR.

In light of the effect of the Y122F mutation in El on its localization, and the lack of effect of the same mutation on its ability to interact with TmaR, we asked whether this mutation affects EI function, by streaking El-mCherry and EI Y122F-mCherry on fructose-supplemented MacConkey plates together with a PTS-KO strain (negative control). The results in Fig. 5G show that sugar consumption is not affected by the $\mathrm{Y} 122 \mathrm{~F}$ mutation in $\mathrm{El}$, suggesting that TmaR phosphorylation on tyrosine is not important for the function of $\mathrm{El}$ in sugar uptake.

Overall our results show that tyrosine phosphorylation of both TmaR and El is important for their polar localization. However, whereas TmaR tyrosine phosphorylation site is important for its interaction with $\mathrm{El}$, the tyrosine phosphorylation site on $\mathrm{El}$ is not important for its interaction with TmaR and, therefore, does not affect its activity. Together, our results suggest that the interaction between the TmaR and $\mathrm{El}$ is the crucial stage in the control El activity as a sugar regulator.

\section{TmaR is important for $E$. coli survival in mild acidic conditions in a sugar- and El-dependent manner}

Our finding that a strain deleted for tmaR consumes glucose faster than a strain carrying the tma $R$ gene calls the role of the TmaR protein into question: under which environmental conditions that $E$. coli encounters does TmaR absence confer a disadvantage?

In nature, E. coli often encounters mild acidic conditions ( $\mathrm{pH} \mathrm{5.0),} \mathrm{e.g.,} \mathrm{in} \mathrm{macrophages,} \mathrm{in} \mathrm{the}$ intestine and in specific foods, or extreme acidic conditions, like in mammalian stomach $(\mathrm{pH} \mathrm{1-3)}$ (34) (35). We, therefore, compared the survival of wild-type and TmaR-KO cells grown at different $\mathrm{pH}$ in minimal media supplemented with glucose. When grown at $\mathrm{pH} 5.5$ in the presence of the most frequently used glucose concentration (0.4\%), TmaR-KO displayed a drastic reduction in 
survival compared to the wild-type strain, as evident by the drop in the number of TmaR-KO cells after 3 days in this medium by four orders of magnitude compared to wild-type (Fig. 6A). The same effect was observed when cells were grown at $\mathrm{pH} 5$ (Fig. 6B, middle panel). However, at pH 7.0 or pH 4 and below, both strains survived similarly or did not survive at all, respectively (Fig. 6B, upper and lower panels, respectively), indicating that only mild acidity causes the observed drop in survival of cells lacking TmaR. The reduction in survival of TmaR-KO cells in acidic medium could be reversed by inoculating the cells into a neutral $\mathrm{pH}$ medium after 3 days, but was irreversible after 4 days (see SI Appendix, Fig. S21).

Because fast transport of glucose is expected to lead to an increase in overflow metabolism to acetate, which acidifies the medium, we wondered if this is the cause for the decreased survival of the TmaR-KO cells. We therefore measured the $\mathrm{pH}$ of several wild-type and TmaR-KO cultures after growth for 3 days at $\mathrm{pH} 5.5$ and observed a small, but highly consistent and reproducible difference: while the $\mathrm{pH}$ in the WT cultures was $\mathrm{pH} 4.1$, that of the TmaR-KO was 4. Hence, the improved glucose uptake by TmaR-KO acidifies the medium faster, compared to WT, and might partly explain the difference in survival.

Monitoring cell survival in $\mathrm{pH} 5.5$ in the presence of $0.4 \%$ glucose at specific time points showed that there was no significant difference in cell survival between the wild-type and TmaR-KO in the first 48 hours of growth (see SI Appendix, Fig. S22). Of note, reduction in cell survival in this medium was also observed for wild-type cells, but only after a week, suggesting that a decrease in cell survival at acidic $\mathrm{pH}$ occurs in wild-type, but the absence of TmaR dramatically increases its rate. The results thus far indicate that TmaR-mediated control of glucose consumption is important for long-term survival of $E$. coli in mild acidic conditions.

To learn more about the survivals phenotype, we viewed the wild-type and TmaR-KO cells that were grown for 3 days in minimal medium, $\mathrm{pH} 5.5$, supplemented with $0.4 \%$ glucose under the microscope. One might have expected the non-growing TmaR-KO cells to undergo lysis, but instead, we observed a most distinct difference between the two strains morphology (Fig. 6C). While the WT cells were small and a bit rounded, a typical morphology of $E$. coli cells in deep SP, the TmaR-KO cells were elongated, resembling more cells in logarithmic than in SP, a surprising morphology for cells that cannot grow on plates, that is, cannot divide successfully. Another difference was the detection of a bright spot near one pole of the TmaR-KO cells, which might represent protein inclusion bodies, lipids inclusions or another dense aggregation. Furthermore, when grown on fructose-supplemented MacConkey plate, the wild-type colonies turned from red to white already after one or two days, while the TmaR-KO colonies remained red even after 3 days (Fig. 6D) and longer (not shown), when the medium was very acidic due to sugar fermentation. Hence, TmaR-KO do not cease consuming sugar in acidic $\mathrm{pH}$, although their survival in these conditions is greatly reduced compared to wild-type cells. Bacteria picked from this plate after 2 or 3 days showed the same difference in morphology observed between wild-type and TmaR-KO cells grown in liquid medium (see SI Appendix, Fig. S23). Hence, it seems that the TmaR-KO cells 
cannot enter SP properly in acidic conditions and accumulate defective molecules, leading to their death in mildly acidic conditions.

The above results raised the question whether the level of the RNA polymerase sigma factor RpoS, the primary regulator of SP genes, changes in TmaR-KO cells. Hence, we compared the level of RpoS in wild-type and TmaR-KO cells grown in acidic or neutral medium for 1, 2 or 3 days by Western blot analysis, using anti-RpoS antibodies. The results (see SI Appendix, Fig. S24) show that, whereas in neutral $\mathrm{pH}$ the level of RpoS increases gradually over time in both strains, in acidic $\mathrm{pH}$, the level of RpoS in wild-type cells decreases after a day, while its level in TmaR-KO cells does not seem to change over time. Hence, reduction in TmaR-KO cells survival in acidic conditions correlates with a higher level of RpoS compared to wild-type cells, maybe hinting at an attempt to enter SP.

Since we observed that absence of TmaR leads to an increase in the kinetics of PTS sugars uptake, we asked whether the concentration of glucose in the acidic medium plays a role in TmaRdependent cell survival. Our results show that when the glucose concentration is reduced from $0.4 \%$ to $0.1 \%$ or to $0.04 \%$, the reduction in TmaR-KO cell survival was less severe. That is, in $0.1 \%$ glucose, the survival of the TmaR-KO cells was somewhat less reduced than in $0.4 \%$ glucose, and no difference was observed between the survivals of the two strains in $0.04 \%$ glucose (Fig. 6A). Increasing the glucose concentration from $0.4 \%$ to $1 \%$ did not make the survival defect of TmaR-KO more severe (Fig. 6A), suggesting that the cells reached saturation in glucose consumption and/or that sugar toxicity is not involved in this phenomenon. Indeed, we observed no elevation in the level of YigL, one of the hallmarks of sugar toxicity, whose level has previously been shown to elevate in sugar stress $(36,37)$, in TmaR-KO compared to WT cells grown at $\mathrm{pH}$ 5.5 in the presence of $0.4 \%$ glucose after 3 and 4 days (see SI Appendix, Fig. S25). Hence, the survival defect of Tma-KO cells in acidic conditions, although influenced by glucose concentration, is not due to sugar toxicity.

Next, we asked if El hyperactivity for sugar consumption in TmaR-KO cells is the cause for the reduced survival in acidic $\mathrm{pH}$. To address this question, we expressed El-mCherry (EI-OE) or only mCherry (CTRL) from an arabinose-induced promoter in TmaR-KO cells and grew them in minimal medium at $\mathrm{pH} 5.5$ with $0.4 \%$ glucose for 3 days. The TmaR-KO cells overexpressing El-mCherry $(0.1 \%$ arabinose) did not survive at all in these mildly acidic conditions as opposed to TmaR-KO cells overexpressing only the mCherry tag (Fig. 6E, middle panel). This inability of the TmaR-KO cells to survive was a direct consequence of El overexpression, since when El expression was not induced (no arabinose), no survival difference was observed between the strains (Fig. 6E, upper panel). Moreover, when growing these two strains at $\mathrm{pH} 7$ with the arabinose inducer, survival of both strains was the same (Fig. 6E, lower panel). These results directly connect between EI activity and survival in acidic $\mathrm{pH}$ and suggest that El hyperactivity in TmaR-KO cells is the mechanism that negatively affects their survival. 
To understand whether the nature of the carbon source is important for the observed TmaRdependent survival, we tested cell survival in the presence of PTS sugars other than glucose (fructose, trehalose or mannitol), whose uptake is directly affected by El the same as glucose, of a non-PTS sugar (lactose), whose uptake is indirectly affected by El (38), and of non-sugar carbon sources (casamino acids (CAA) or succinate) that are not known to be affected by EI and are incorporated into the bacterial carbon metabolism differently than sugars (39). When cells were grown in the presence of fructose and trehalose, TmaR-KO displayed a reduction in cell survival at 72 hours, similar to that observed with glucose; in the presence of mannitol, the reduction in cell survival was less severe; and in the presence lactose, CAA, and succinate, there was no change in survival compared to wild-type (Fig. 6F). However, after a week of growth, a severe defect in TmaR-KO survival was observed with mannitol and lactose, but not with CAA and succinate (Fig. 6G), suggesting that a drop in survival occurs in the presence of carbon sources that can enter glycolysis - PTS and non-PTS sugars - albeit at different rates, but not with non-sugar carbon sources. Taken together, our results suggest that TmaR-mediated regulation of sugar consumption (PTS or non PTS) is important for cell survival in acidic environments.

Finally, to figure out if the inability of TmaR to get phosphorylated on $\mathrm{Y} 72$ also affects survival in acidic conditions, we compared survival in $\mathrm{pH} 5.5$ of cells expressing TmaR Y72F, TmaR Y79F and TmaR triple mutant to that of cells expressing wild-type TmaR and TmaR-KO cells. The results show that, unlike TmaR deletion, impairment of TmaR phosphorylation had no effect on survival in acidic conditions(see SI Appendix, Fig. S26), in line with our finding that TmaR deletion and TmaR inability to get phosphorylated had opposite effects on sugar consumption.

\section{DISCUSSION}

How biochemical reactions are compartmentalized within a bacterial cell is largely an open question. Unlike eukaryotes, which have organelles that enable them to control their enzymatic functions within a restricted environment, most prokaryotes do not have membrane-bounded organelles. Obviously, bacteria have developed strategies to efficiently process biochemical reactions in their crowded cytoplasm, but their nature is mostly unknown. Here, we describe a hitherto unrecognized layer of regulation of sugar metabolism in bacteria, which is based on the spatiotemporal organization of the genera PTS protein EI. We discovered TmaR, a novel protein, which localizes to the pole depending on its phosphorylation on a tyrosine residue, and show that it regulates sugar metabolism by controlling the activity of El via polar sequestration and release, thus enabling survival in mild acidic conditions (schematically illustrated in Fig. 7).

TmaR is the first $E$. coli protein reported to localize to the poles depending on its phosphorylation on a tyrosine residue. A SILAC-based analysis of the $E$. coli phosphoproteome predicted that TmaR is phosphorylated on TyR 79 (21), rather than on Tyr 72, as indicated by our results. A possible explanation for this discrepancy is that the proximity of the two residues enables 
phosphorylation of both, which depends on Tyr 72 being phosphorylation first. Indeed, a careful look at TmaR Y79F probed with anti-pTyr (Fig. 1G) reveals a slight reduction in the intensity of the band compared to WT. Phosphorylation on multiple residues, depending on the conditions has often been observed in eukaryotes (e.g., (40) (41) (42)). An alternative explanation is that TmaR is phosphorylated on different tyrosine residues depending on the growth phase and the environmental conditions. Indeed, phosphorylation on Tyr79 was detected during SP (21), whereas our results show that less TmaR molecules localize to the poles in this phase. Remarkably, all three tyrosines residues in TmaR are preceded by an aspartic acid, culminating in a DY motif, which is one of 13 statistically significant sequence motifs predicted for tyrosine phosphorylation sites in human proteins (27). Analysis of 512 tyrosine phosphorylation sites in $E$. coli showed underrepresentation of lysine residues in position $+2(27)$, which is the case for TyR 55 , making Tyr72 and Tyr79 more favorable sites for phosphorylation. Intriguingly, the sequence around Tyr 79 matches one of the five motifs, YXXK, identified for bacterial proteins phosphorylated on tyrosine (27). Future research will hopefully elucidate the role of Tyr79 phosphorylation.

The kinase that phosphorylates TmaR is not known. Despite of the numerous proteins predicted to be phosphorylated on tyrosines in E. coli, only two BY kinases, Wzc and Etk, are known in this organism, and they both contain two transmembrane domains and are involved in cell envelope maintenance $(25,26)$. The observation that deletion of both wzc and etk genes did not diminish the extent of tyrosine phosphorylation in $E$. coli cells (27) indicates that additional tyrosine kinases await to be discovered in this organism.

We have previously suggested that the poles serve as hubs for sensory systems, thus enabling their communication and the generation of optimal responses (16). We have recently shown that RNAs that are involved in sensing and responding also accumulate at the poles (5). TmaRmediated sequestration of El molecules at the pole, highlight the importance of poles as depots that enable the production of key factors before they are needed and their release upon need, providing an additional level of regulation of sugar consumption. Such storage mechanisms have been previously described in E. coli for MurG and FtsZ $(8,9)$, but the proteins contributing to their polar deposition are not known. Regulation of the sugar regulator Mlc by the glucose permease PtsG has been shown to occur via membrane sequestration (43). Of note, tyosine phosphorylation-dependent recruitment of pole localizers in other bacteria has not been reported, although phosphorylation of DivIVA on a serine in B. subtilis, (44) and on a threonine in Streptococcus suis (45) were detected, but direct evidence for their occurrence in vivo has not been obtained yet, let alone for their involvement in polar localization.

TmaR is usually observed in one cell pole, unless overexpressed. Of note, the two poles in organisms that divide symmetrically, such as E. coli and B. subtilis, are different, one old and the other new. Various events which may potentially occur in both poles, end up taking place only in one, such as cell division during sporulation of $B$. subtilis (46) or plasmolysis in E. coli (47). Polar proteins often localize to one pole in these organisms, when expressed from the chromosome, 
although their overexpression reveals that the two poles have capacity to retain them. Other proteins were shown to oscillate from pole to pole or to relocate to midcell, which is the pole to be $(15,48)$. Future experiments with the microfluidic mother machine $(49)$ are required to determine which proteins have a preference for a certain pole, old or new.

Our data showing that TmaR and El interact in vivo and in vitro provides a mechanism for TmaRmediated polar clustering of El. This concept is indirectly supported by our findings that when TmaR is overexpressed, more El molecules accumulate at the pole, whereas when $\mathrm{El}$ is overexpressed, less TmaR molecules localize at the pole. Thus, assembly of the two proteins at the pole is controlled by a stoichiometric balance between them. This stoichiometry does not reflect the ratio between the total number of molecules of each of these proteins in the cell and can, therefore, be affected by changing the level of one of them. Changing the balance affects sugar consumption, as evidenced by the increased sugar consumption when TmaR is absent and $\mathrm{El}$ is, therefore, non-clustered. This stoichiometric balance suggests that rather than functioning as an ON-OFF switch, TmaR functions as a fine-tuner that regulates El activity by controlling the dynamics of El clustering and release. For such a mechanism to be efficient, the interactions between the two proteins need to be weak and easily reversible (typical of phase separation, see below). Our results suggest that this type of interaction depends on TmaR phosphorylation on Tyr 72 , since substitution of this residue to a non-phosphorylatable residue renders the interaction quite irreversible and prevents EI release from the pole. To the best of our knowledge, this is the first report of a protein, which is not a part of the PTS family that has a global effect on the PTS pathway by spatiotemporally controlling its major regulator.

We previously suggested that factors mediating El polar clustering localize to membrane regions with strong negative curvature (14). However, sequence analysis does not predict any membranetraversing or -binding motifs in TmaR. Additionally, our previous (15) and present results show that both El and TmaR are dynamic, mainly within the pole region and do not appear to be membraneanchored, suggesting that their localization involves additional mechanisms, such as the formation of condensates via liquid-liquid phase separation (LLPS) (50). Evidence supporting this notion is the FRAP analysis of EI that shows the recruitment of EI molecules into the cluster in few minutes (15), which might indicate high fluidity of the cluster, characteristic of LLPS (51). Moreover, both TmaR and El are predicted to have unstructured domains (52), a common feature of proteins capable of LLPS (51). Also tyrosine phosphorylation, shown here to be important for TmaR and EI localization, has been implicated in LLPS in eukaryotes, as it enables electrostatic interactions that mediate LLPS (53). Because TmaR controls El localization, but not vice versa, TmaR is expected to be the one to induce the putative LLPS. Our finding that TmaR limits the degree of heterogeneity in El activity is in line with this hypothesis, since compartmentalization of proteins by LLPS has been suggested as a mechanism to reduce noise (54). Further research to examine this possibility is required. 
The phosphorylated tyrosine residue in El, Tyr122, is a conserved residue between $\mathrm{El}^{\text {sugar }}$ and $\mathrm{EI}^{\mathrm{Ntr}}$ (33), suggestive of its importance. Notably, extensive tyrosine phosphorylation occurs in proteins linked to sugar metabolism and the TCA cycle in E. coli and in the archaeon Sulfolobus solfataricus (55), suggesting that the impact of tyrosine phosphorylation on proteins that function in carbohydrate metabolism is conserved through evolution. The finding that tyrosine phosphorylation regulates the activity of the catabolite regulator Cra (22), together with our findings that it regulates localization of El and of the factor that controls El activity, TmaR, supports this hypothesis. The finding that among the proteins that co-purified with TmaR, the biggest group was of proteins that are involved in metabolic processes strengthen this assumption.

The multifaceted effect of $\mathrm{TmaR}$ on regulating sugar metabolism and cell survival at low $\mathrm{pH}$ explains why TmaR is a conserved protein. Although TmaR is not essential in laboratory growth conditions, it is apparently essential for bacterial survival in their natural habitats, e.g., the human colon ( $\mathrm{pH}$ 6-7.5), the caecum, which is the beginning of the large intestine ( $\mathrm{pH} 5.7$ ) and the small intestine ( $\mathrm{pH} 4-6)$ (56), as well as macrophages $(\mathrm{pH}$ 5.0) (34). As for sugars, glucose concentration in the human colon is estimated to reach up to $40 \mathrm{mM}$ (57), but food intake and digestion lead to rapid changes in gut sugar concentrations. Hence, counteracting the stress associated with high sugar concentration and low $\mathrm{pH}$ is particularly essential in the gut. We suggest that TmaR makes an important regulatory connection between sugar uptake and cell survival in mild acidic conditions, since survival of TmaR-KO cells under these conditions is observed only when the cells are grown with sugars and not with other carbon sources. This might be explained by the production of lactate or pyruvate and/or due to El phosphorylation by PEP that produces pyruvate, both due to the sugars that enter glycolysis (59). Overproduction of pyruvate or its acidic derivatives can lower the $\mathrm{pH}$ and create harmful conditions that eventually might kill the cells and, therefore, require acid resistance (57). Importantly, overexpression of El reduces survival in mildly acidic conditions just like deletion of TmaR, strengthening our assumption that this effect is mediated through EI hyperactivity. Because EI and the PTS in general play roles in many regulatory pathways, such as carbon catabolite repression, nitrogen and phosphate metabolism, potassium transport and chemotaxis $(58,59)$, the effect of TmaR on El activity is expected to have a far bigger effect on cell physiology than just the control of sugar flux.

Why do cells lacking TmaR die in mild acidic $\mathrm{pH}$ ? Our results argue against the possibility that they are more susceptible to sugar toxicity. On the other hand, the morphology of the TmaR-KO cells after growing them for 3 days at pH 5.5 suggest that they cannot properly enter SP, a phase that enables cells to adapt to and survive in suboptimal environments that involve nutrient deprivation. Entry into SP requires complex and coordinated changes in gene expression, which enable reduction in cell metabolism and energy conservation. Because bacteria spend most of their time in nature in stressful conditions and intense competition for resources, difficulties in entering SP is a severe defect that makes cells waste their limited resources, much like "let us eat and drink, for 
tomorrow we shall die" (Isaiah 22). This is exemplified by the red TmaR-KO colonies after 3 days on MacConkey plates, as opposed to wild-type colonies that turn completely white.

The alternative sigma factor RpoS controls the SP-related changes in gene expression, allowing cells to become more resistant not only to the stress that they first encounter but also to other stressful treatments $(60,61)$. The report on the increase in RpoS levels in various stresses (62), which reflects the need to combat with stressful conditions might explain the elevation in RpoS level in TmaR-KO cells compared to wild-type in acidic conditions. The linkage between the control of rpoS expression and carbon metabolism suggests that the uncontrolled activity of El in TmaR$\mathrm{KO}$ cells affects proper entry into SP. Of note, expression of tmaR is dramatically increased in $\Delta r p o S$ cells (63), suggesting that control of TmaR level is important for survival in SP.

\section{MATERIALS AND METHODS}

All methods and materials used for this study are described in the Supporting Information. This includes constructions of all strains, fluorescence microscopy and image analysis, biochemical methodologies used for protein analysis and protein-protein interaction and microbial practices.

\section{ACKNOWLEDGMENTS}

We thank Avital Cher for screening the YFP-tagged ORFs for their localization, Omer Goldberger for help with the illustration and Mikel Irastorza for critical reading of the manuscript. We acknowledge Joachim O. Radler and Abram Aertsen for the gift of T1683 and IbpA-msfGFP strains, respectively. We are grateful to Susan Gottesman, Teppei Morita and Hiroji Aiba for the generous gift of $\alpha-R p o S$ and $\alpha{ }_{I I E B}{ }^{G l c}$ antibodies, as well as to Ophry Pines and Sigal Ben-Yehuda for sharing antibodies against His-tag and GFP-tag, respectively. We thank Reuven Wiener for help with the $\mathrm{pET}$ plasmids, Yair Katz for help with Matlab and Excel, Meshi Barsheshet for help with analysis with R, and Yair E. Gatt for generating TmaR phylogenetic tree. We thank members of Orna Amster-Choder and Hanah Margalit labs for fruitful discussions and Regine Hengge and Roberto Kolter for consultation about RpoS. This research was supported by the Israel Science Foundation (ISF) founded by the Israel Academy of Sciences and Humanities (1274/19) and the Deutsch-Israeli Project Cooperation (DIP) (AM 441/1-1 SO 568/1-1).

\section{REFERENCES}

1. C. Gauthier-Rouviere, M. Vandromme, D. Tuil, N. Lautredou, M. Morris, M. Soulez, A. Kahn, A. Fernandez, N. Lamb, Expression and activity of serum response factor is required for expression of the muscle-determining factor MyoD in both dividing and differentiating mouse C2C12 myoblasts. Mol. Biol. Cell. 7, 719-729 (1996).

2. D. Z. Rudner, R. Losick, Protein subcellular localization in bacteria. Cold Spring Harb. Perspect. Biol. 2, a000307 (2010).

3. K. Nevo-Dinur, A. Nussbaum-Shochat, S. Ben-Yehuda, O. Amster-Choder, Translationindependent localization of mRNA in E. coli. Science (80-. ). 331, 1081-1084 (2011). 
bioRxiv preprint doi: https://doi.org/10.1101/2020.08.01.232603; this version posted August 4,2020 . The copyright holder for this preprint (which was not certified by peer review) is the author/funder. All rights reserved. No reuse allowed without permission.

4. L. Shapiro, H. H. McAdams, R. Losick, Why and how bacteria localize proteins. Science. 326, 1225-8 (2009).

5. S. Kannaiah, J. Livny, O. Amster-choder, Spatiotemporal organization of the E. coli transcriptome: Translation-independence and engagement in regulation. Mol. cell Press (2019).

6. S. Govindarajan, O. Amster-Choder, Where are things inside a bacterial cell? Curr. Opin. Microbiol. 33 (2016), pp. 83-90.

7. I. V Surovtsev, C. Jacobs-Wagner, Subcellular Organization: A Critical Feature of Bacterial Cell Replication. Cell. 172, 1271-1293 (2018).

8. A. M. Michaelis, Z. Gitai, Dynamic polar sequestration of excess MurG may regulate enzymatic function. J. Bacteriol. 192, 4597-4605 (2010).

9. J. Yu, Y. Liu, Z. Chang, An Organelle-like Structure Correlated with the Quiescent State of Bacterial Cells. bioRxiv (2017).

10. H. Fukuoka, Y. Inoue, A. Ishijima, Coordinated regulation of multiple flagellar motors by the Escherichia coli chemotaxis system. Biophysics (Oxf). 8 (2012), pp. 59-66.

11. J. Deutscher, F. M. D. Aké, M. Derkaoui, A. C. Zébré, T. N. Cao, H. Bouraoui, T. Kentache, A. Mokhtari, E. Milohanic, P. Joyet, The bacterial phosphoenolpyruvate:carbohydrate phosphotransferase system: regulation by protein phosphorylation and phosphorylationdependent protein-protein interactions. Microbiol. Mol. Biol. Rev. 78, 231-56 (2014).

12. M. H. Saier, The Bacterial Phosphotransferase System: New Frontiers 50 Years after Its Discovery. J. Mol. Microbiol. Biotechnol. 25 (2015), pp. 73-78.

13. L. Lopian, Y. Elisha, A. Nussbaum-Shochat, O. Amster-Choder, Spatial and temporal organization of the E. coli PTS components. EMBO J. 29, 3630-3645 (2010).

14. S. Govindarajan, Y. Elisha, K. Nevo-Dinur, O. Amster-Choder, The general phosphotransferase system proteins localize to sites of strong negative curvature in bacterial cells. MBio. 4 (2013), doi:10.1128/mBio.00443-13.

15. S. Govindarajan, N. Albocher, T. Szoke, A. Nussbaum-Shochat, O. Amster-Choder, Phenotypic heterogeneity in sugar utilization by $\mathrm{E}$. coli is generated by stochastic dispersal of the general PTS protein El from polar clusters. Front. Microbiol. 8 (2018), doi:10.3389/fmicb.2017.02695.

16. O. Amster-Choder, The compartmentalized vessel: The bacterial cell as a model for subcellular organization (a tale of two studies). Cell. Logist. 1, 77-81 (2011).

17. C. Tropini, K. C. Huang, Interplay between the Localization and Kinetics of Phosphorylation in Flagellar Pole Development of the Bacterium Caulobacter crescentus. PLoS Comput. Biol. 8, 1002602 (2012).

18. R. T. Wheeler, L. Shapiro, Differential localization of two histidine kinases controlling bacterial cell differentiation. Mol. Cell. 4, 683-694 (1999).

19. P. Casino, V. Rubio, A. Marina, The mechanism of signal transduction by two-component systems. Curr. Opin. Struct. Biol. 20 (2010), pp. 763-771.

20. Y. Taniguchi, P. J. Choi, G. W. Li, H. Chen, M. Babu, J. Hearn, A. Emili, X. Sunney Xie, Quantifying E. coli proteome and transcriptome with single-molecule sensitivity in single cells. Science (80-. ). 329, 533-538 (2010).

21. N. C. Soares, P. Spät, K. Krug, B. MacEk, Global dynamics of the Escherichia coli proteome and phosphoproteome during growth in minimal medium. J. Proteome Res. 12, 2611-2621 (2013).

22. C. D. Robertson, T. H. Hazen, J. B. Kaper, D. A. Rasko, A. M. Hansen, Phosphotyrosinemediated regulation of enterohemorrhagic Escherichia coli virulence. MBio. 9 (2018), doi:10.1128/mBio.00097-18.

23. Z. Chen, P. A. Cole, Synthetic approaches to protein phosphorylation. Curr. Opin. Chem. 
bioRxiv preprint doi: https://doi.org/10.1101/2020.08.01.232603; this version posted August 4,2020 . The copyright holder for this preprint (which was not certified by peer review) is the author/funder. All rights reserved. No reuse allowed without permission.

Biol. 28 (2015), pp. 115-122.

24. G. Lazer, L. Pe'er, M. Farago, K. Machida, B. J. Mayer, S. Katzav, Tyrosine residues at the carboxyl terminus of Vav1 play an important role in regulation of its biological activity. J. Biol. Chem. 285, 23075-23085 (2010).

25. C. Vincent, P. Doublet, C. Grangeasse, E. Vaganay, A. J. Cozzone, B. Duclos, Cells of Escherichia coli contain a protein-tyrosine kinase, Wzc, and a phosphotyrosine-protein phosphatase, Wzb. J. Bacteriol. 181, 3472-3477 (1999).

26. O. Ilan, Y. Bloch, G. Frankel, H. Ullrich, K. Geider, I. Rosenshine, Protein tyrosine kinases in bacterial pathogens are associated with virulence and production of exopolysaccharide. EMBO J. 18, 3241-3248 (1999).

27. A.-M. Hansen, R. Chaerkady, J. Sharma, J. J. Díaz-Mejía, N. Tyagi, S. Renuse, H. K. C. Jacob, S. M. Pinto, N. A. Sahasrabuddhe, M.-S. Kim, B. Delanghe, N. Srinivasan, A. Emili, J. B. Kaper, A. Pandey, The Escherichia coli Phosphotyrosine Proteome Relates to Core Pathways and Virulence. PLoS Pathog. 9, e1003403 (2013).

28. J. Deutscher, C. Francke, P. W. Postma, How Phosphotransferase System-Related Protein Phosphorylation Regulates Carbohydrate Metabolism in Bacteria. Microbiol. Mol. Biol. Rev. 70, 939-1031 (2006).

29. E. Gabor, A. K. Göhler, A. Kosfeld, A. Staab, A. Kremling, K. Jahreis, The phosphoenolpyruvate-dependent glucose-phosphotransferase system from Escherichia coli K-12 as the center of a network regulating carbohydrate flux in the cell. Eur. J. Cell Biol. 90 (2011), pp. 711-720.

30. Y. Tanaka, K. Kimata, H. Aiba, A novel regulatory role of glucose transporter of Escherichia coli: membrane sequestration of a global repressor Mlc. EMBO J. 19, 5344-5352 (2000).

31. S. A. Westermayer, G. Fritz, J. Gutiérrez, J. A. Megerle, M. P. S. WeißI, K. Schnetz, U. Gerland, J. O. Rädler, Single-cell characterization of metabolic switching in the sugar phosphotransferase system of Escherichia coli. Mol. Microbiol. 100, 472-485 (2016).

32. S. K. Govers, J. Mortier, A. Adam, A. Aertsen, Protein aggregates encode epigenetic memory of stressful encounters in individual Escherichia coli cells. PLoS Biol. 16, e2003853 (2018).

33. M. Strickland, A. M. Stanley, G. Wang, I. Botos, C. D. Schwieters, S. K. Buchanan, A. Peterkofsky, N. Tjandra, Structure of the NPr:EINNtr Complex: Mechanism for Specificity in Paralogous Phosphotransferase Systems. Structure. 24, 2127-2137 (2016).

34. A. Majumdar, D. Cruz, N. Asamoah, A. Buxbaum, I. Sohar, P. Lobel, F. R. Maxfield, Activation of microglia acidifies lysosomes and leads to degradation of Alzheimer amyloid fibrils. Mol. Biol. Cell. 18, 1490-1496 (2007).

35. J. Lin, M. P. Smith, K. C. Chapin, H. S. Baik, G. N. Bennett, J. W. Foster, Mechanisms of acid resistance in enterohemorrhagic Escherichia coli. Appl. Environ. Microbiol. 62, 3094100 (1996).

36. Y. Sun, C. K. Vanderpool, Physiological consequences of multiple-target regulation by the small RNA SgrS in escherichia coli. J. Bacteriol. 195, 4804-4815 (2013).

37. K. Papenfort, Y. Sun, M. Miyakoshi, C. K. Vanderpool, J. Vogel, Small RNA-mediated activation of sugar phosphatase mRNA regulates glucose homeostasis. Cell. 153, 426-437 (2013).

38. R. Bruckner, F. Titgemeyer, Carbon catabolite repression in bacteria: choice of the carbon source and autoregulatory limitation of sugar utilization. FEMS Microbiol. Lett. 209, 141-148 (2002).

39. K. Y. Alam, D. P. Clark, Anaerobic fermentation balance of Escherichia coli as observed by in vivo nuclear magnetic resonance spectroscopy. J. Bacteriol. 171, 6213-7 (1989).

40. C. Gowda, C. Song, Y. Ding, S. Iyer, P. K. Dhanyamraju, M. McGrath, Y. Bamme, M. Soliman, S. Kane, J. L. Payne, S. Dovat, Cellular signaling and epigenetic regulation of 
gene expression in leukemia. Adv. Biol. Regul. 75 (2020), , doi:10.1016/j.jbior.2019.100665.

41. J. A. M. J. L. Janssen, New Insights from IGF-IR Stimulating Activity Analyses: Pathological Considerations. Cells. 9 (2020), , doi:10.3390/cells9040862.

42. M. Fabbro, B. B. Zhou, M. Takahashi, B. Sarcevic, P. Lal, M. E. Graham, B. G. Gabrielli, P. J. Robinson, E. A. Nigg, Y. Ono, K. K. Khanna, Cdk1/Erk2- and Plk1-dependent phosphorylation of a centrosome protein, Cep55, is required for its recruitment to midbody and cytokinesis. Dev. Cell. 9, 477-488 (2005).

43. S. J. Lee, W. Boos, J. P. Bouché, J. Plumbridge, Signal transduction between a membranebound transporter, PtsG, and a soluble transcription factor, Mlc, of Escherichia coli. EMBO J. 19, 5353-5361 (2000).

44. V. Ravikumar, L. Shi, K. Krug, A. Derouiche, C. Jers, C. Cousin, A. Kobir, I. Mijakovic, B. Macek, Quantitative phosphoproteome analysis of Bacillus subtilis reveals novel substrates of the kinase PrkC and phosphatase PrpC. Mol. Cell. Proteomics. 13, 1965-1978 (2014).

45. H. Ni, W. Fan, C. Li, Q. Wu, H. Hou, D. Hu, F. Zheng, X. Zhu, C. Wang, X. Cao, Z. Q. Shao, $X$. Pan, Streptococcus suis DivIVA protein is a substrate of Ser/Thr kinase STK and involved in cell division regulation. Front. Cell. Infect. Microbiol. 8 (2018), doi:10.3389/fcimb.2018.00085.

46. A. Feucht, I. Lucet, M. D. Yudkin, J. Errington, Cytological and biochemical characterization of the FtsA cell division protein of Bacillus subtilis. Mol. Microbiol. 40, 115-125 (2001).

47. E. Mulder, C. L. Woldringh, Plasmolysis bays in Escherichia coli: Are they related to development and positioning of division sites? J. Bacteriol. 175, 2241-2247 (1993).

48. G. Laloux, C. Jacobs-Wagner, How do bacteria localize proteins to the cell pole? J. Cell Sci. 127, 11-9 (2014).

49. P. Wang, L. Robert, J. Pelletier, W. L. Dang, F. Taddei, A. Wright, S. Jun, Robust growth of escherichia coli. Curr. Biol. 20, 1099-1103 (2010).

50. S. Boeynaems, S. Alberti, N. L. Fawzi, T. Mittag, M. Polymenidou, F. Rousseau, J. Schymkowitz, J. Shorter, B. Wolozin, L. Van Den Bosch, P. Tompa, M. Fuxreiter, Protein Phase Separation: A New Phase in Cell Biology. Trends Cell Biol. 28 (2018), pp. 420-435.

51. S. Alberti, A. Gladfelter, T. Mittag, Considerations and Challenges in Studying Liquid-Liquid Phase Separation and Biomolecular Condensates. Cell. 176 (2019), pp. 419-434.

52. O. Paliy, S. M. Gargac, Y. Cheng, V. N. Uversky, A. Dunker, Protein disorder is positively correlated with gene expression in escherichia coli. J. Proteome Res. 7, 2234-2245 (2008).

53. I. Owen, F. Shewmaker, The role of post-translational modifications in the phase transitions of intrinsically disordered proteins. Int. J. Mol. Sci. 20 (2019), , doi:10.3390/ijms20215501.

54. A. Klosin, F. Oltsch, T. Harmon, A. Honigmann, F. Jülicher, A. A. Hyman, C. Zechner, Phase separation provides a mechanism to reduce noise in cells. Science (80-. ). 367, 464468 (2020).

55. L. J. Getz, C. S. Runte, J. K. Rainey, N. A. Thomas, Tyrosine phosphorylation as a widespread regulatory mechanism in prokaryotes. J. Bacteriol. 201 (2019), , doi:10.1128/JB.00205-19.

56. J. Lin, M. P. Smith, K. C. Chapin, H. S. U. K. Baik, G. N. Bennett, J. W. Foster, Mechanisms of Acid Resistance in Enterohemorrhagic Escherichia coli. 62, 3094-3100 (1996).

57. P. L. Moreau, The lysine decarboxylase CadA protects Escherichia coli starved of phosphate against fermentation acids. J. Bacteriol. 189, 2249-2261 (2007).

58. A. Galinier, J. Deutscher, Sophisticated Regulation of Transcriptional Factors by the Bacterial Phosphoenolpyruvate: Sugar Phosphotransferase System. J. Mol. Biol. 429, 773789 (2017).

59. J. Deutscher, M. Désirée, M. Derkaoui, C. Zébré, N. Cao, The Bacterial Phosphoenolpyruvate : Carbohydrate Phosphotransferase System : Regulation by Protein 
Phosphorylation and Phosphorylation-. 78, 231-256 (2014).

60. E. R. Zinser, R. Kolter, Escherichia coli evolution during stationary phase. Res. Microbiol. 155 (2004), pp. 328-336.

61. A. Battesti, N. Majdalani, S. Gottesman, The RpoS-Mediated General Stress Response in Escherichia coli . Annu. Rev. Microbiol. 65, 189-213 (2011).

62. M. J. Mandel, T. J. Silhavy, Starvation for different nutrients in Escherichia coli results in differential modulation of RpoS levels and stability. J. Bacteriol. 187, 434-442 (2005).

63. A. Ito, T. May, K. Kawata, S. Okabe, Significance of rpoS during maturation of Escherichia coli biofilms. Biotechnol. Bioeng. 99, 1462-1471 (2008).

\section{FIGURE LEGENDS}

Figure 1. TmaR forms clusters that localize to the pole of $E$. coli cells in a tyrosine phosphorylation-dependent manner.

(A) Images of cells expressing TmaR-YFP (yellow) from its native promoter and locus in the chromosome. Cells were grown in $L B$ to $\mathrm{OD}_{600}=0.2$. Cells are in phase (gray).

(B) The fluorescence intensity profile of 50 cells expressing a cluster of TmaR-YFP along the normalized long cell axis. Each cell is presented by a different color and the average is shown by a bold black line. YFP intensity is in arbitrary units.

(C) Percent of cells at the indicated $O D_{600}$ that showed a detectable cluster in the population. Calculation was made with the strain expressing TmaR-YFP grown in LB ( $N=500)$. The bars show the standard deviation between three biological repeats.

(D) Western blot analysis showing the level of TmaR-YFP in cells at the indicated $O_{600}(O N$, cells grown overnight) and of GroEL (control used for normalization) detected by a-mCherry and $\alpha$ GroEL antibodies, respectively.

(E) Images of cells expressing YFP-tagged TmaR (WT) and its variants mutated in each of its three tyrosines, (Y79F, Y72F and Y51F), in two of its tyrosines (Y79F,Y72F) and in all three (Y79F,Y72F,Y51F), all expressed from TmaR native promoter and locus in the chromosome. Cells are in phase (gray).

(F) The average fraction of cells with clusters of TmaR derivatives in each strain. WT; TmaR Y79F; TmaR Y51F, TmaR Y79F, Y72F and TmaR Y79F,Y72F,Y51F. The bars show the standard deviations between different fields $(\mathrm{N}=250)$.

(G) Western blot analysis of cells expressing YFP-tagged TmaR (WT) or its variants mutated in each of the three tyrosines, (Y79F, Y72F and Y51F), in two of the tyrosines (Y79F,Y72F) and in all three tyrosines $(\mathrm{Y} 79 \mathrm{~F}, \mathrm{Y} 72 \mathrm{~F}, \mathrm{Y} 51 \mathrm{~F})$, all from the $\mathrm{pET15b}$ vector, as well as cells with no plasmid (NC, negative control). The membrane was probed with anti-phosphorylated tyrosine antibody.

Figure 2. TmaR and El exhibit co-localization and co-dynamics and are capable of interacting with each other 
(A) Images of cells expressing El-mCherry (red) and TmaR-YFP (yellow), both expressed from their native promoter and locus in the chromosome. Cells are in phase (gray).

(B) Heat map showing the localization of El-mCherry (red) and TmaR-YFP (yellow). The color intensity represents the strength of the fluorescent signal in the same cell along the long cell axis (arbitrary units, AU). Each lane represents a single cell $(\mathrm{N}=100)$. Because of the heterogeneity in cells length, the area that is devoid of cell body is in grey.

(C) Upper panels: Single-cell kymograph of the merged TmaR-YFP and El-mCherry (orange), TmaR-YFP (yellow) and El-mCherry (red). The kymograph X-axis is time (0-12 min) and the Y-axis is the cell length along its long axis. Lower panels: The cell (outlined) shown in the kymograph.

(D) TmaR-YFP and El- mCherry clusters were monitored in 163 cells every 3 minutes for 3 hours by time-lapse microscopy. Each row represents one cell over time. Orange, both TmaR and El clusters are present in the cell; yellow, only the TmaR cluster is present in the cell; grey, no cluster is present in the cell.

(E) Far-Western analysis of the interaction between $\mathrm{El}$ and TmaR. Lysates of $\Delta$ tmaR cells expressing the His-tag only (lanes 1 ) or His-tagged TmaR (lanes 2) were analyzed by SDS-PAGE, blotted onto a nitrocellulose membrane and probed with: anti-His antibody (I), El-mCherry followed by anti-mCherry antibody (II) or mCherry followed by anti-mCherry antibody (III). The observed bands match His-TmaR size ( 15 kDa).

(F) Mass spectrometry analysis showing the fold change of El co-purification on anti-GFP antibody-bound resin from cells endogenously expressing TmaR-YFP vs. cells endogenously expressing non-tagged TmaR (WT). The pts/ gene codes for the El protein.

(G) Volcano plot presentation of the mass spectrometry results. The number of peptides identified for each protein was plotted against the log fold change in this protein intensity between cells expressing TmaR-YFP and cells expressing non-tagged TmaR (WT). The El (Ptsl) and TmaR are depicted as blue dots. A red dotted lines show the values for $\mathrm{El}$.

(H) A pie chart showing the biological processes, identified by GO analysis, in which the proteins identified as significantly co-purifying with TmaR by mass spectrometry are involved.

\section{Figure 3. TmaR is essential for El polar localization, but not vice versa}

(A) From left to right: Images showing El-mCherry in wild-type cells (WT), in cells deleted for the tmaR gene (TmaR-KO), in wild-type cells overexpressing TmaR (TmaR-OE) and in TrmaR-KO cells overexpressing TmaR (TmaR-KO+OE).

(B) The average fraction of cells with El clusters in the different strains: WT, TmaR-KO and TmaR$\mathrm{OE}(\mathrm{N}=500)$. The bars show the standard deviation between different fields.

(C) The mean of the sum intensity of the El-mCherry clusters (arbitrary units, AU): WT and TmaR$\mathrm{OE}(\mathrm{N}=464)$. The bars show the standard deviation between different fields. 
(D) Western blot analysis showing the level of El-mCherry and GroEL proteins (control used for normalization) detected by $\alpha-m C h e r r y$ and $\alpha$-GroEL antibodies, respectively.

(E) Images showing TmaR-YFP localization in wild-type cells (WT, left panel) and in cells deleted for the pts operon that encodes EI (PTS-KO, right panel). TmaR-YFP was expressed from its native promoter and locus in the chromosome. Cells are in phase (grey). (F) The average fraction of cells with TmaR clusters in WT and PTS-KO strain $(\mathrm{N}=400)$. The bars show the standard deviations between different fields.

(G) Images showing the localization of TmaR-YFP, expressed from the chromosome, in wild-type cells (WT, left panel) and in cells overexpressing EI (EI-OE).

(H) Images of wild-type cells (WT, left panel) and cells deleted for tmaR (TmaR-KO, right panel) overexpressing HPr-GFP. Cells are in phase (grey).

Figure 4. TmaR-mediated El localization affects sugar uptake by the PTS system

(A) A representative MacConkey plate supplemented with $0.4 \%$ fructose showing the phenotype of wild-type cells (WT), cells deleted for the tmaR gene (TmaR-KO), wild-type cells overexpressing TmaR (TmaR-OE) and cells deleted for the pts operon (PTS-KO).

(B) The rate of glucose consumption by the following strains: WT (blue), TmaR-KO (red) TmaR-OE (green), and PTS-KO (purple). The bars show the standard deviation between three biological repeats.

(C) The rate of glucose consumption in mmoles per min for the WT (blue), TmaR-KO (red) and TmaR-OE (green). The bars show the standard deviation between three biological repeats.

(D) A scatter plot showing the mean intensity (arbitrary units, AU) of mCerulean expressed from $P_{\text {NAG }}$ as a function of the cellular El-mCherry mean intensity (AU) after 1 hour of transition to NAGcontinuing medium for WT cells (blue $\mathrm{Xs}, \mathrm{N}=103$ ) and TmaR-KO strain (red $\mathrm{Xs}, \mathrm{N}=103$ ). The $r$ squared $\left(R^{2}\right)$ values and Pearson correlation coefficients $(\rho)$ are given.

Figure 5. The effect of replacement of TmaR and El tyrosines on their interaction and on EI localization and activity.

(A) Far-Western analysis of the interaction between purified El and TmaR (WT) or its variants mutated in each of the three tyrosines, (Y79F, Y72F and Y51F), in two of the tyrosines $(\mathrm{Y} 79 \mathrm{~F}, \mathrm{Y72F})$ and in all three tyrosines (Y79F,Y72F,Y51F), all expressed from plasmids as Histagged proteins in a $\Delta t m a R$ background, as well as lysates of $\Delta t m a R$ cells expressing only a Histag or no protein from plasmids as controls (NC, negative control). Equal amount of lysates were fractionated by SDS-PAGE and blotted onto two nitrocellulose membranes, one incubated with anti-His antibodies (Western, a) and the other with purified mCherry-tagged El followed by 
antibodies against mCherry (Far Western, b). The detected band is at TmaR expected size ( 15 $\mathrm{kDa})$. The intensity of the bands detected in the Far Western analysis was normalized to the intensity of the respective bands detected in the Western blot analysis (c).

(B) Images of cells showing localization of El-mCherry in cells overexpressing wild-type GFPTmaR (TmaR) or non-phosphorylated GFP-TmaR (TmaR Y72F).

(C) Images of cells expressing El-mCherry and IbpA-msfGFP in cells overexpressing non-tagged wild-type TmaR (TmaR) or non-phosphorylated TmaR (TmaR Y72F).

(D) A representative MacConkey plate supplemented with $0.4 \%$ fructose and $0.1 \mathrm{mM}$ IPTG showing the phenotype of $\triangle t m a R$ cells overexpressing TmaR (WT) and its mutants with the indicated substitutions of TmaR tyrosines, all expressed from the lac promoter in pCA24N, as well as of $\Delta t m a R(T m a R-K O)$ cells and cells deleted for the pts operon (PTS-KO)..

(E) Images of cells expressing EI-mCherry and El Y122F-mCherry, both expressed from the ptsI promoter and locus in the chromosome. Cells are in phase (gray).

(F) Far-Western analysis of the interaction between purified EI Y122F-mCherry and His-tagged TmaR in a $\Delta t m a R$ background. The details are as described in Fig. 5A for wild-type El. The proteins were blotted onto two nitrocellulose membranes, one incubated with anti-His antibodies (Western, left panel) and the other with purified mCherry-tagged EI Y122F followed by antibodies against mCherry (Far Western, right panel).

(G) A representative MacConkey plate supplemented with $0.4 \%$ fructose showing the phenotype of cells expressing El-mCherry and EI Y122F-mCherry from ptsl promoter and locus on the chromosome and of cells deleted for the pts operon (PTS-KO).

Figure 6. Cells lacking TmaR exhibit a drastic reduction in survival in mildly acidic conditions

(A) Pictures of wild-type (WT) and TmaR-KO (KO) colonies grown in acidic M9 medium (pH 5.5) supplemented with $0.04 \%, 0.1 \%, 0.4 \%$ and $1 \%$ glucose for 72 hours, spotted after serial dilutions on LB plates.

(B) Pictures of wild-type (WT) and TmaR-KO (KO) colonies grown in acidic media adjusted to $\mathrm{pH} 7$ or in neutral M9 adjusted to $\mathrm{pH} 5$ or 4 , all supplemented with $0.4 \%$ glucose, grown for 72 hours and spotted after serial dilutions.

(C) Images of WT and TmaR-KO cells that grew in acidic M9 medium (pH 5.5) supplemented with $0.4 \%$ glucose for 72 hours. Cells are in phase.

(D) A representative MacConkey plate showing the phenotype of cells expressing wild-type TmaR (WT) or TmaR-KO and of cells deleted for the pts operon (PTS-KO) after 3 days incubation. 
(E) Pictures of wild-type colonies overexpressing El-mCherry (EI-OE) or just mCherry (CTRL) grown in acidic $\mathrm{M9}(\mathrm{pH} 5.5)$ or in neutral $\mathrm{M9}(\mathrm{pH}$ 7) supplemented with $0.4 \%$ glucose with or without $0.1 \%$ of arabinose inducer for 72 hours and spotted after serial dilutions.

(F) Pictures of wild-type (WT) and TmaR-KO (KO) cells grown in acidic M9 medium ( $\mathrm{pH}$ 5.5) supplemented with the indicated carbon sources for 72 hours and spotted after serial dilutions as in $(A)$.

(G) Pictures of wild-type (WT) and TmaR-KO (KO) cells grown in acidic M9 medium ( $\mathrm{pH}$ 5.5) supplemented with the indicated carbon sources for one week and spotted after serial dilutions.

Figure 7. A model for TmaR-mediated control of El localization and its implications.

In wild-type cells (WT), tyrosine-phosphorylated TmaR sequesters a fraction of El protein, which is tyrosine-phosphorylated, in polar clusters, thus restricting El activity and enabling survival in mild acidic conditions. In $\Delta t m a R$ cells, $\mathrm{El}$ is completely diffused, enabling hyper-consumption of sugar that is detrimental to bacterial cell survival in acidic conditions. 
Figure 1

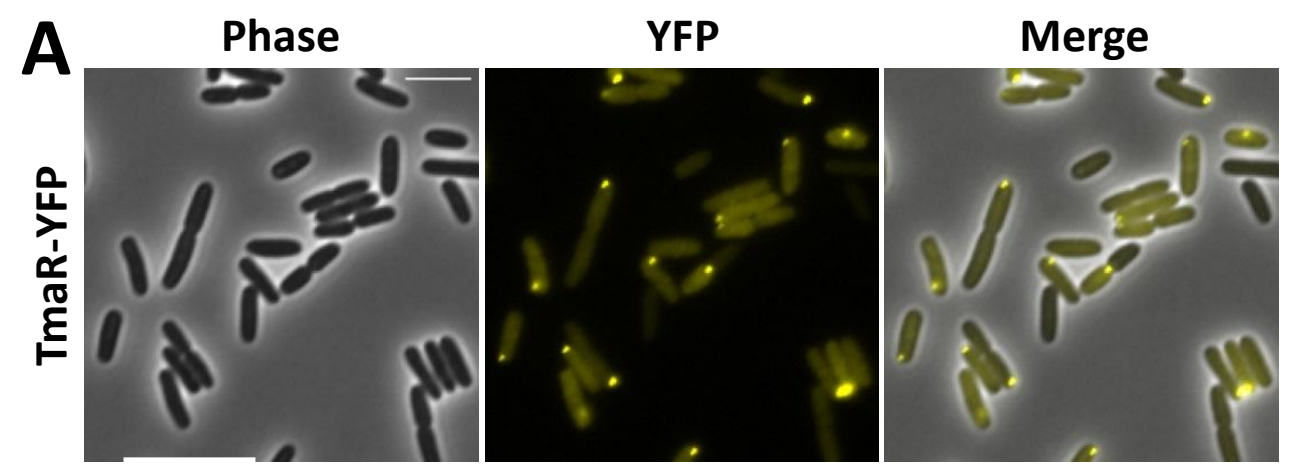

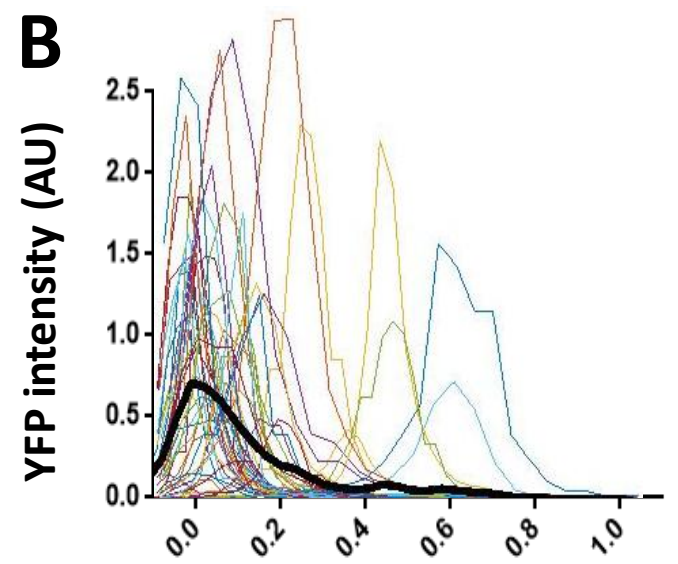

The normalized cell length
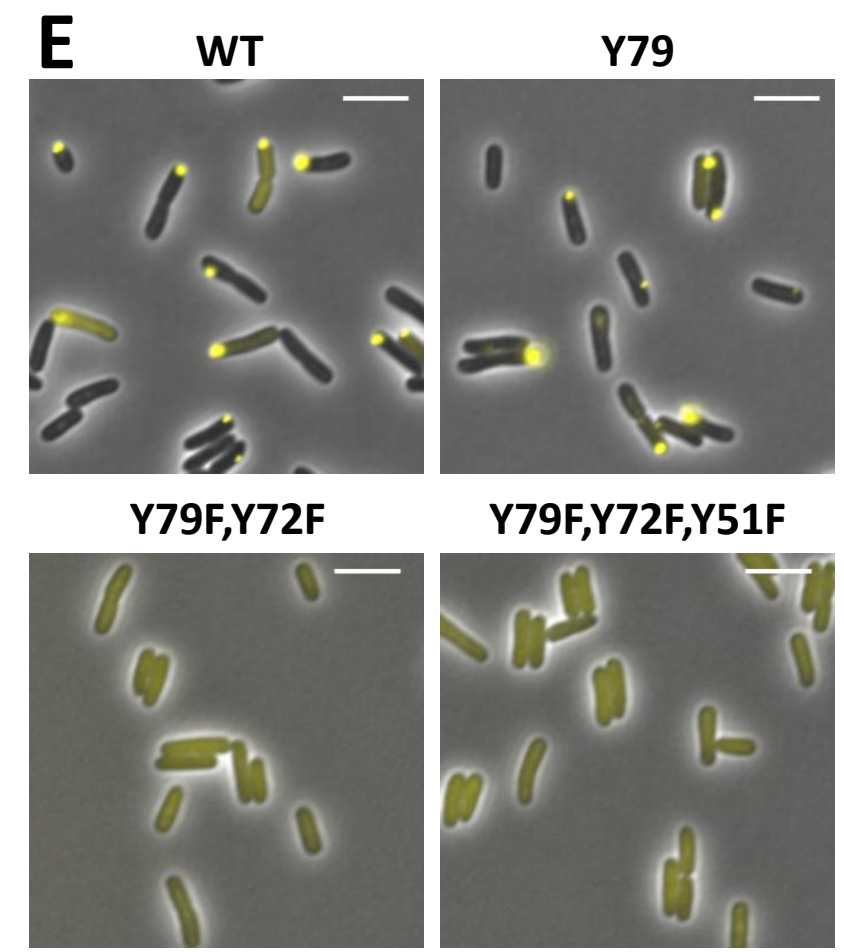

Y79F

$\begin{array}{llll}\mathbf{G} \quad & & & \text { Y79F Y72F } \\ \text { WT } \quad \text { Y79F Y72F } & \text { Y51F } & \text { Y72F Y51F NC }\end{array}$

C

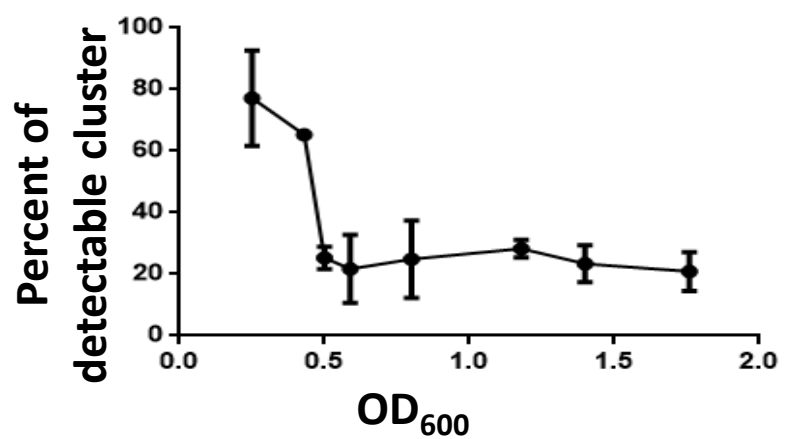

$\begin{array}{llllllllll}D & \mathrm{OD}_{600} & 0.2 & 0.4 & 0.6 & 0.8 & 1 & 1.3 & 1.7 & \mathrm{ON}\end{array}$ TmaR-YFP GroEL

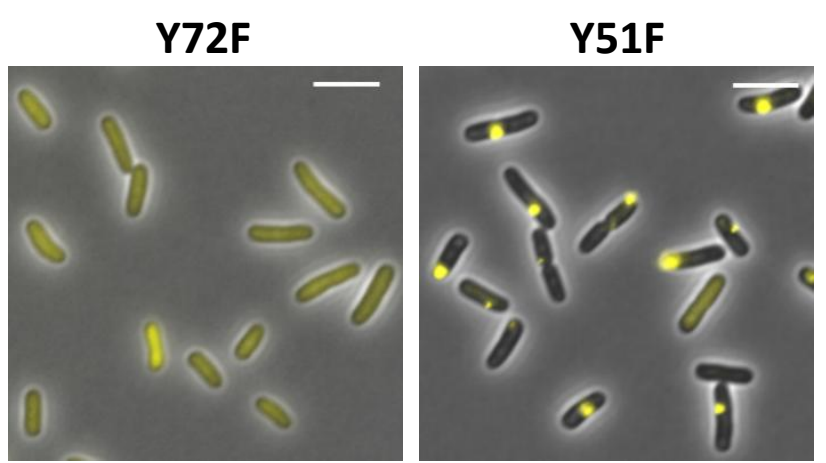

F Fraction of cells with clusters

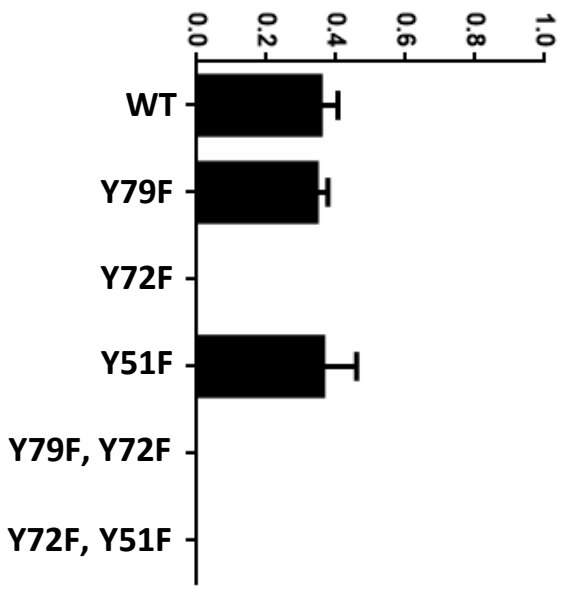




\section{Figure 2}

A Phase
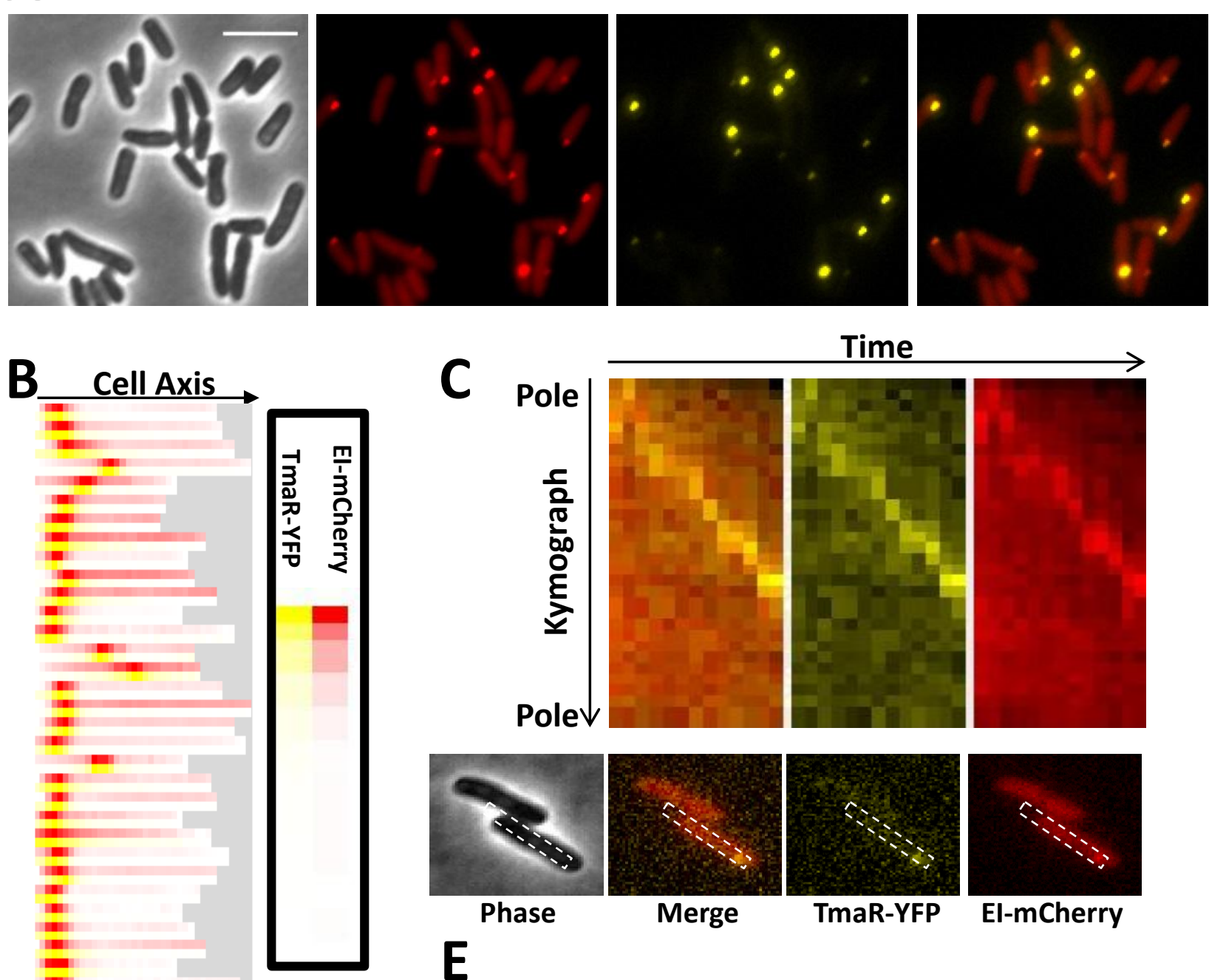

Time
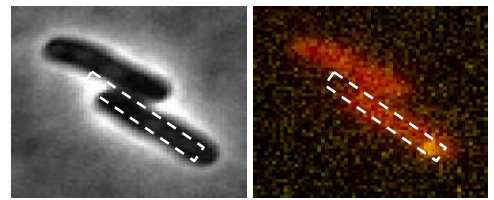

Merge

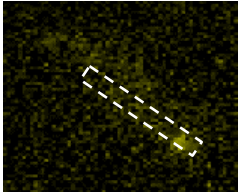

TmaR-YFP

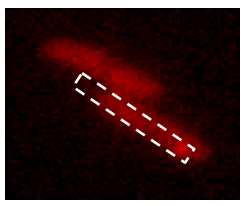

E

Blot reacted with:

I. Anti His

In gel His Hislysates OE: Tag TmaR

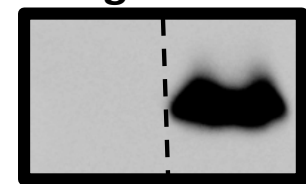

1

II.

Anti mCherry
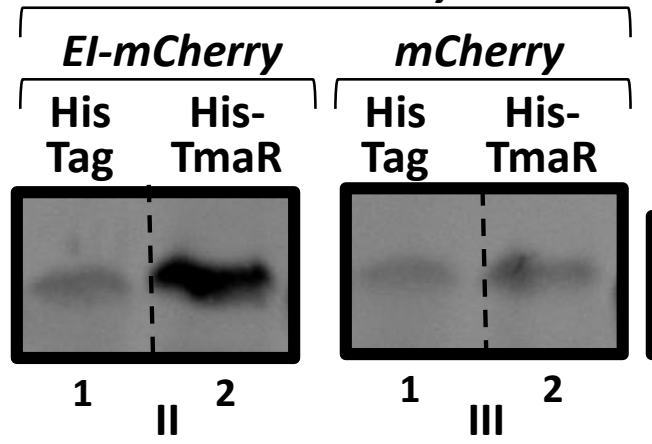

Clusters of TmaR \& EI Cluster of TmaR only No cluster \begin{tabular}{cccc}
\hline Gene & Fold change & $\begin{array}{c}\text { intensity } \\
\text { WT }\end{array}$ & $\begin{array}{c}\text { intensity } \\
\text { TmaR-YFP }\end{array}$ \\
\hline ptsl & $\mathbf{6 . 3}$ & $4.56 \mathrm{E}+05$ & $2.85 \mathrm{E}+06$
\end{tabular}

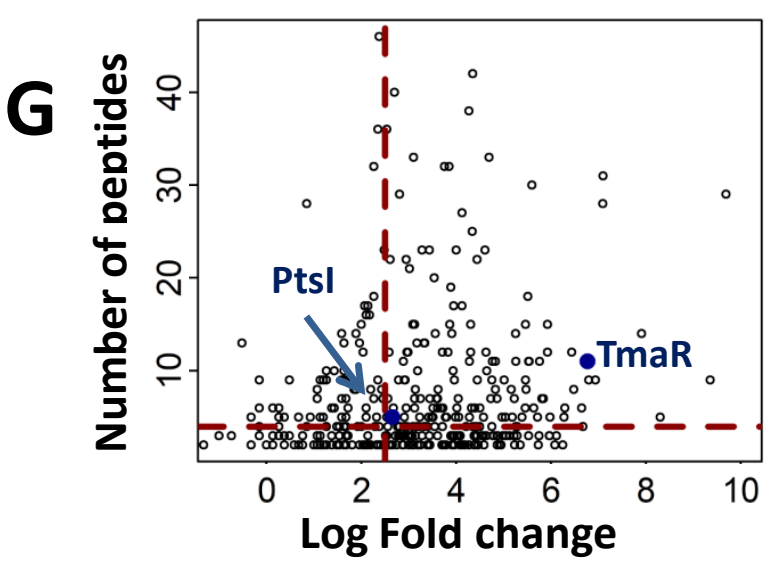

H

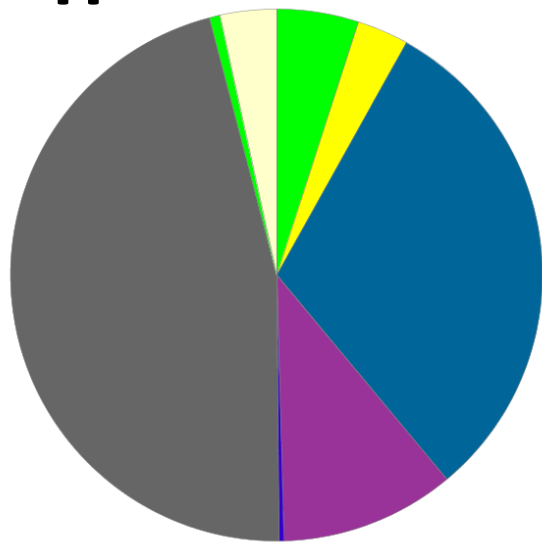

Biological regulation

Cellular component

- Cellular process

- Localization

Locomotion

Metabolic process

Reproduction

Response to stimulus 
Figure 3
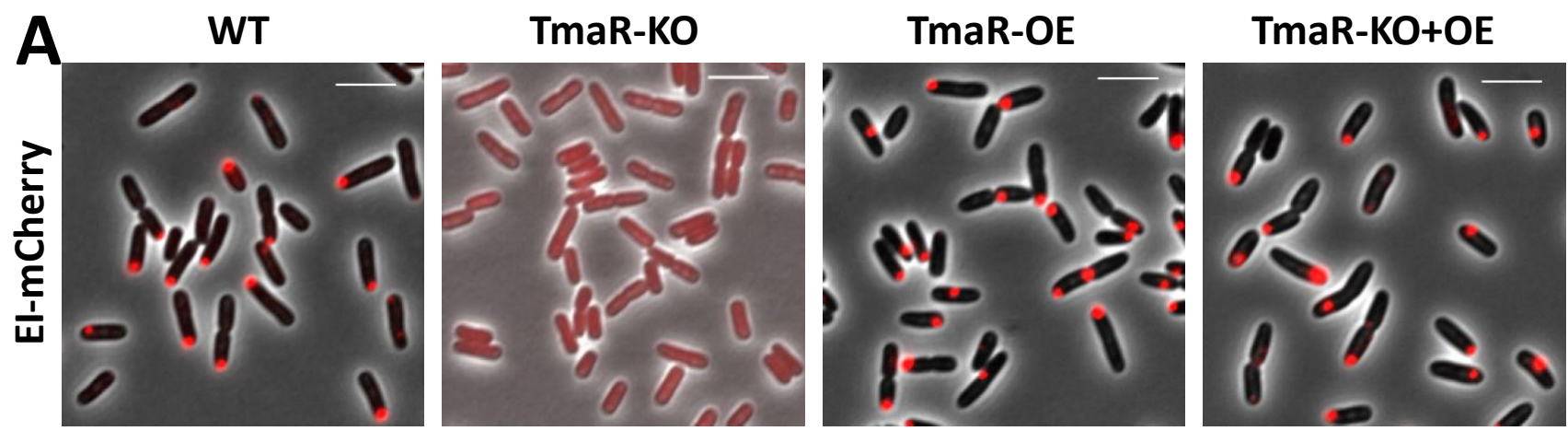

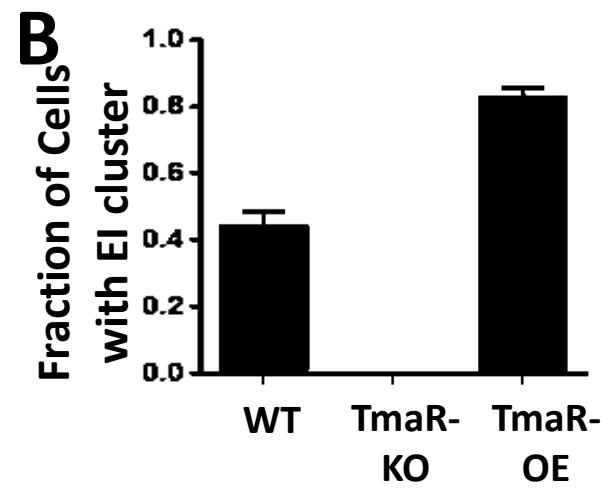

C

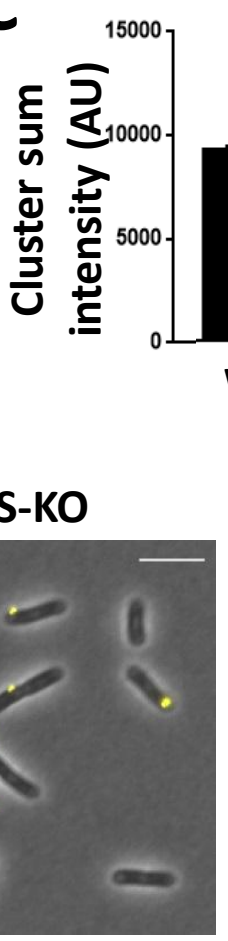

E
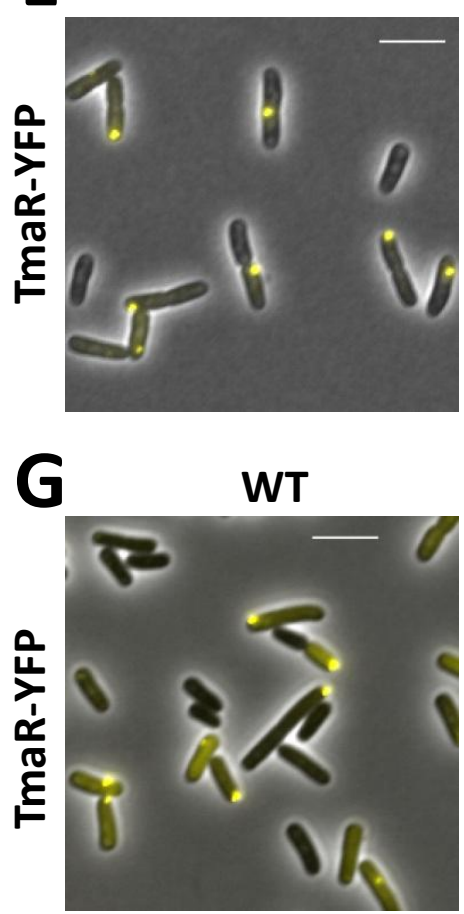

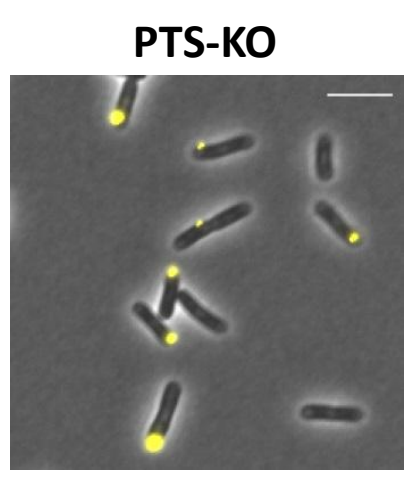

EI-OE

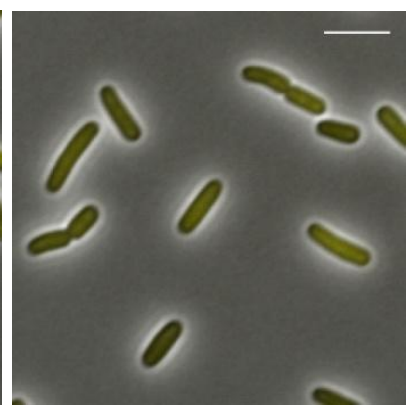

D WT TmaR- TmaRKO OE

EImCherry

GroEL WT TmaROE F
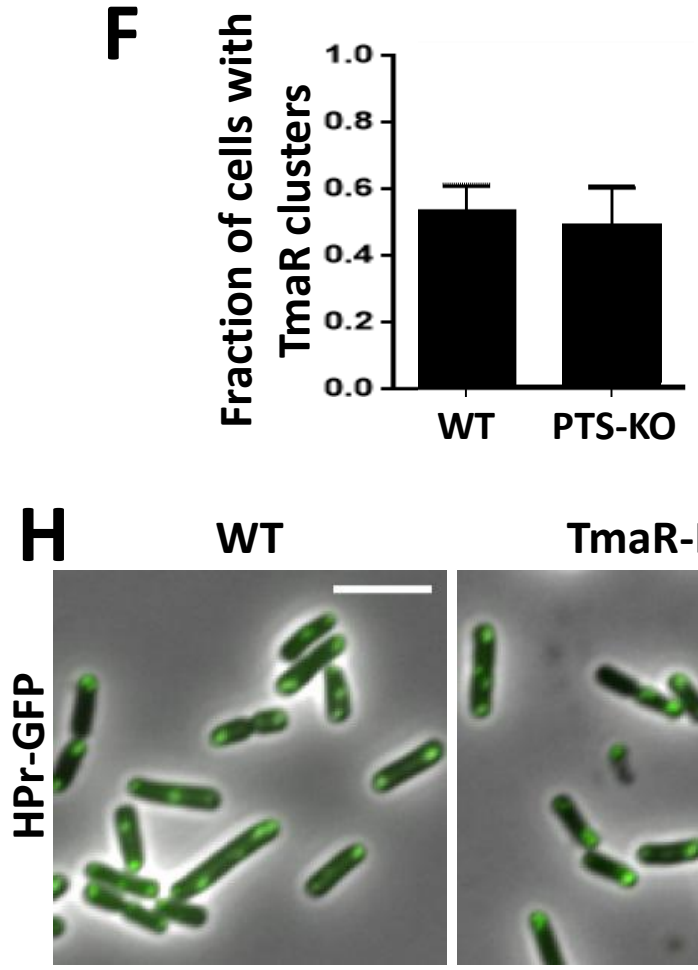
TmaR-KO

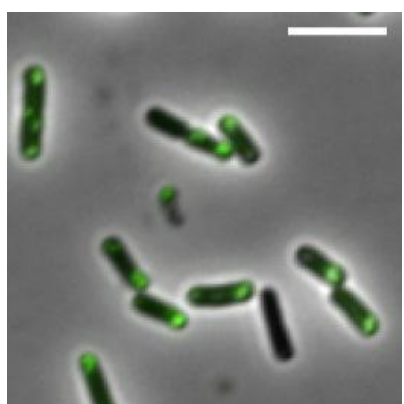


Figure 4

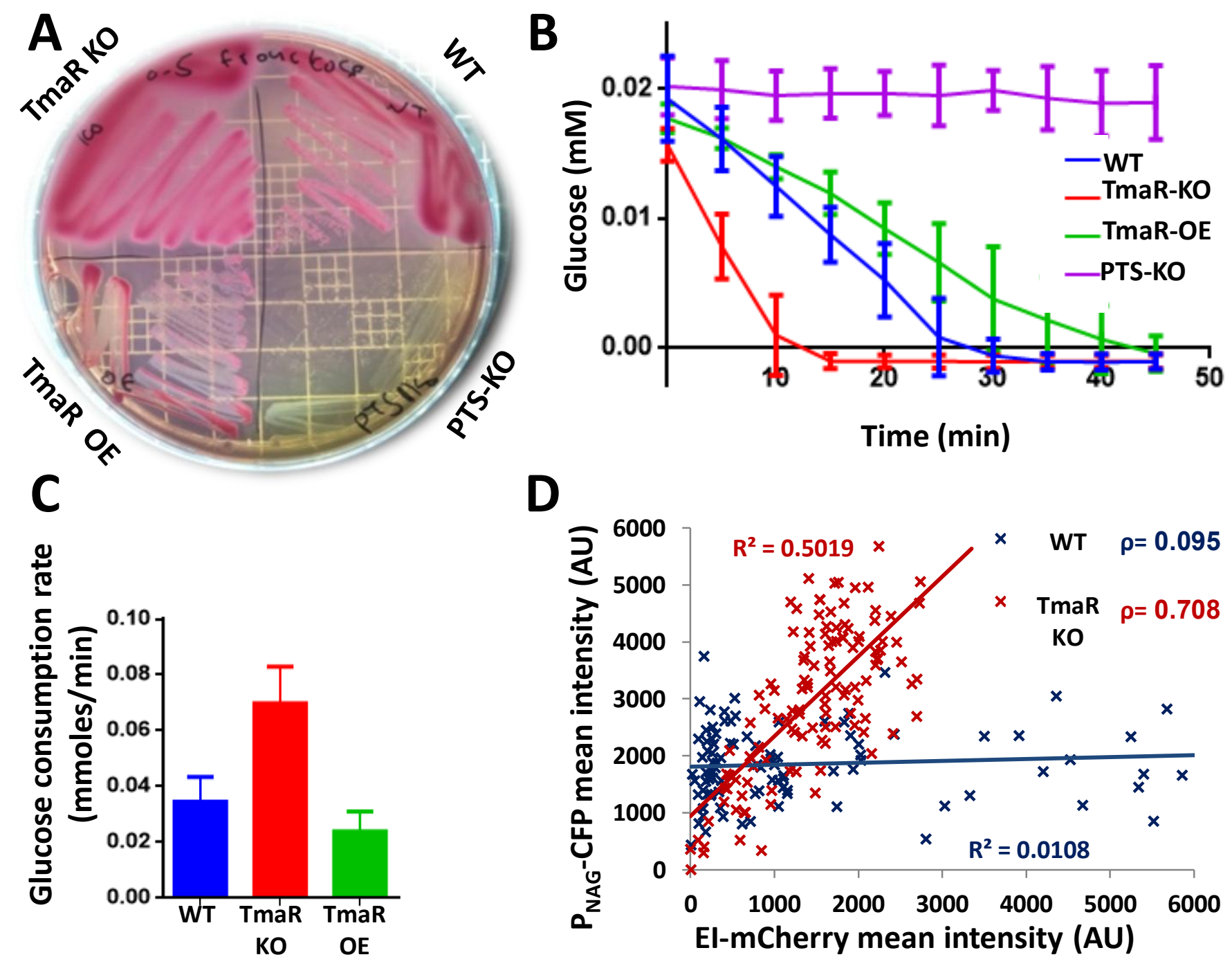


Figure 5

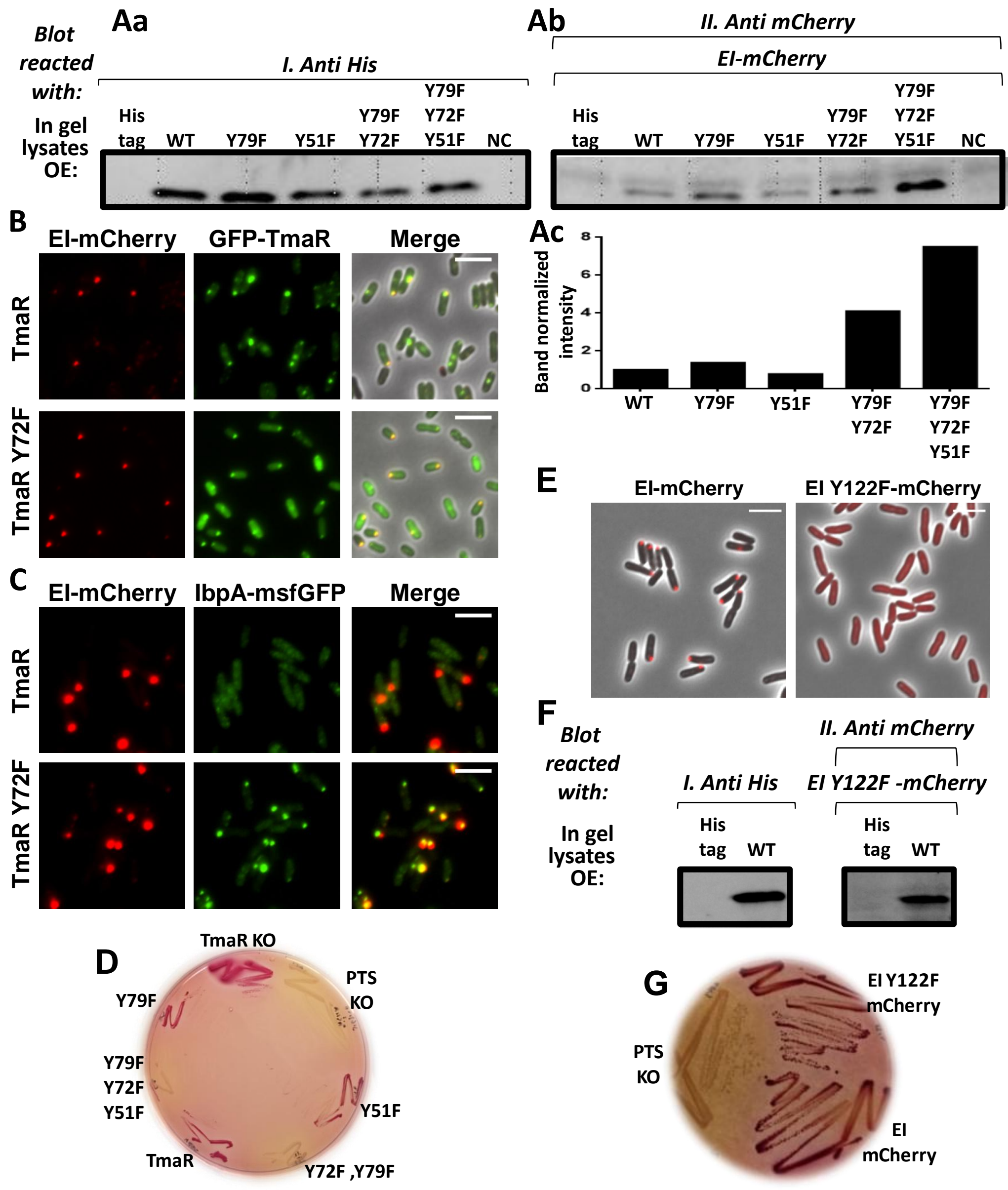


A

WT

KO

WT

KO

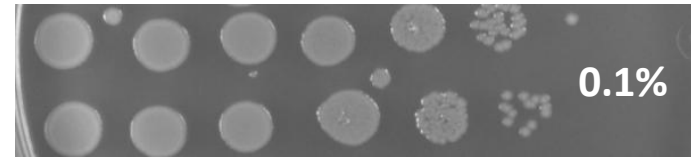

WT

KO

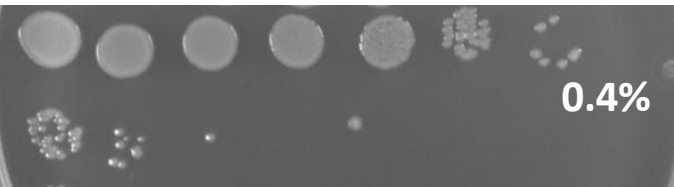

WT

KO

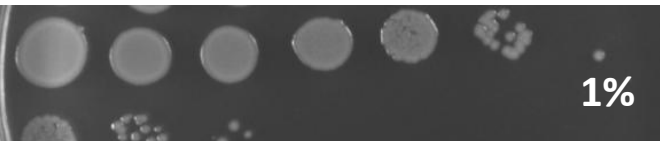

C
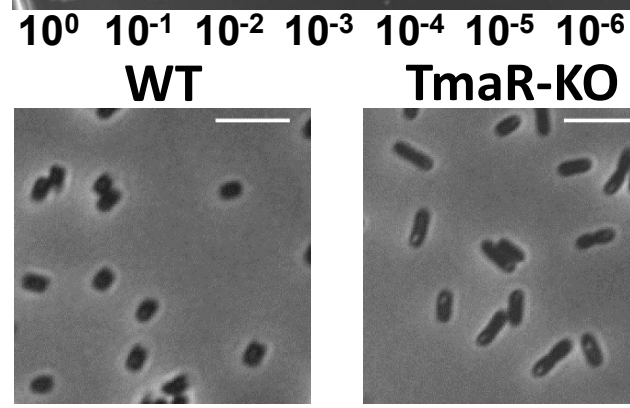

E

CTRL

EI-OE

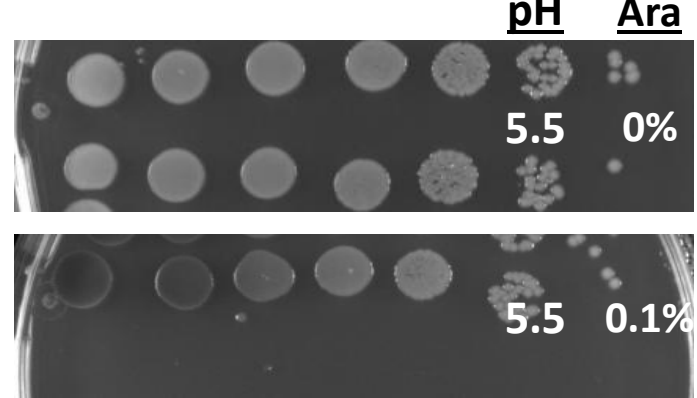

CTRL

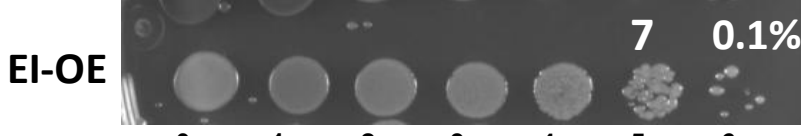

G

$10^{0} \quad 10^{-1} \quad 10^{-2} \quad 10^{-3} \quad 10^{-4} \quad 10^{-5} \quad 10^{-6}$

W

KO

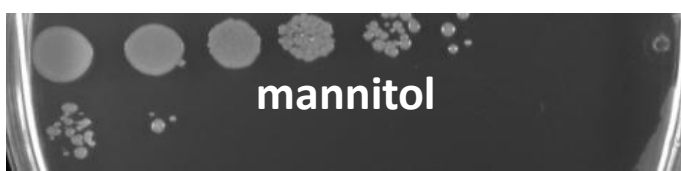

WT

$\mathrm{KO}$

lactose

WT

KO

CAA

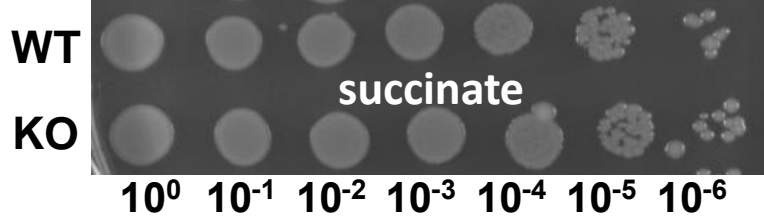

pH

KO
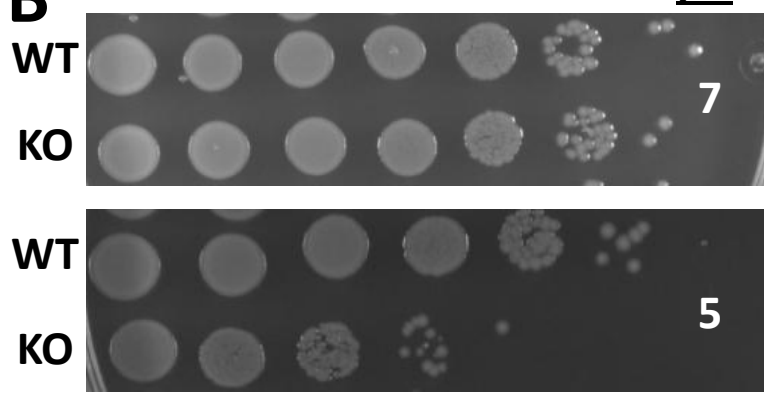

WT

KO

$10^{0} 10^{-1} \quad 10^{-2} \quad 10^{-3} \quad 10^{-4} \quad 10^{-5} \quad 10^{-6}$

D

TmaR

PTS

KO

KO

$\mathbf{F}$

WT

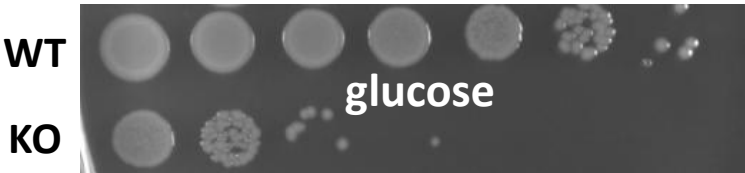

WT

KO

fructose

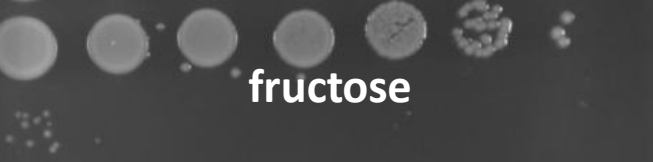

WT

KO

trehalose

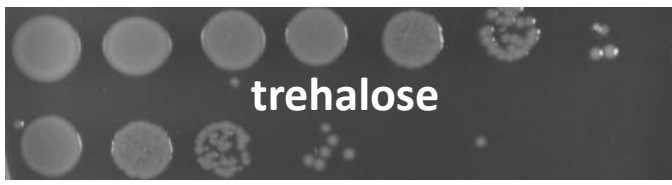

WT

KO

mannitol
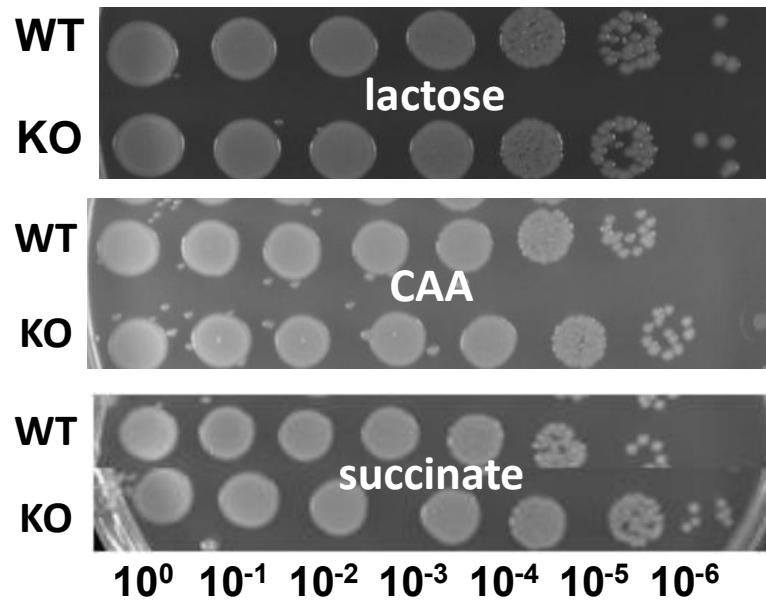
Figure 7

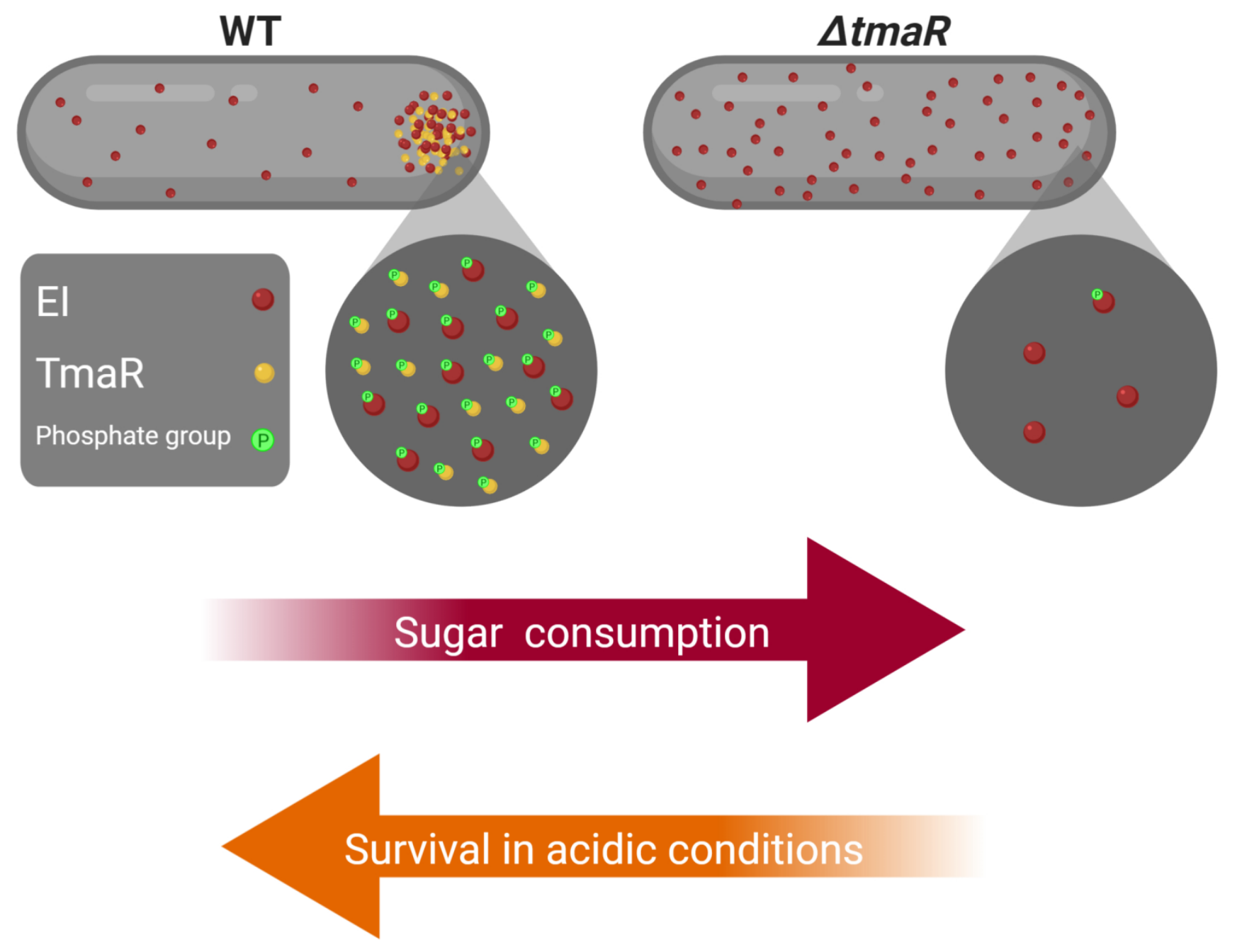

